\title{
A Preliminary Study to Assess Model Uncertainties in Fluid Flows
}

Marc Oliver Delchini Jean C. Ragusa

September 2009

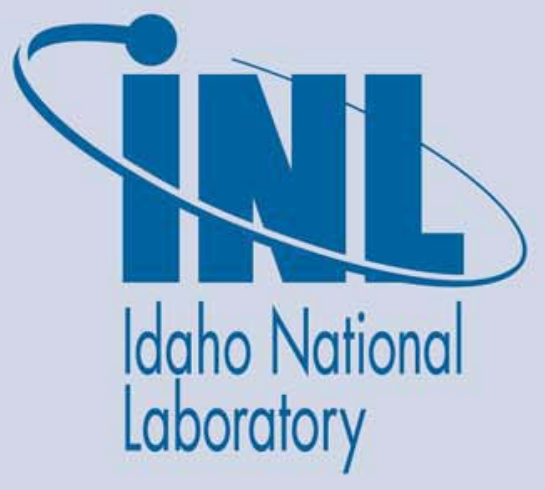

The INL is a U.S. Department of Energy National Laboratory operated by Battelle Energy Alliance 
INL/EXT-09-16995

\title{
A Preliminary Study to Assess Model Uncertainties in Fluid Flows
}

\author{
Marc Oliver Delchini
}

Jean C. Ragusa

September 2009

\begin{abstract}
Idaho National Laboratory
Idaho Falls, Idaho 83415
\end{abstract}

http://www.inl.gov

Prepared for the

U.S. Department of Energy

Through the INL LDRD Program

Under DOE Idaho Operations Office

Contract DE-AC07-05ID14517 


\title{
A Preliminary Study to Assess Model Uncertainties in Fluid Flows
}

\author{
Marc Oliver Delchini \\ Jean C. Ragusa
}

September 29, 2009 


\section{Contents}

1 Introduction 3

2 Geometrical and mathematical models 4

2.1 Model geometry . . . . . . . . . . . . . . . . . . . . 4

2.2 Assumptions for the model . . . . . . . . . . . . . 5

2.2.1 Compressible and incompressible flows . . . . . . . . 7

2.2 .2 Boussinesq correction . . . . . . . . . . . . . 7

2.3 Fluid flow equations with wall friction, gravity, pump and exter-

nal energy source terms . . . . . . . . . . . . . 8

2.3.1 Primitive variables . . . . . . . . . . . . . 9

2.3.2 The continuity equation and momentum equation . . . . 9

2.3.3 The energy equation . . . . . . . . . . . . . 10

2.4 Pump and pressurizer models . . . . . . . . . . . . . . . 11

2.4.1 Pump model . . . . . . . . . . . . . . . . . . . . . 11

2.4.2 Pressurizer model . . . . . . . . . . . . . . . . . . . 11

2.5 Fluid flow models . . . . . . . . . . . . . . . . . . . . 12

2.5.1 Laminar and turbulent flows . . . . . . . . . . . 12

2.5.2 Darcy's law . . . . . . . . . . . . . . . . . . . . 13

2.6 Final forms of the equations . . . . . . . . . . . . . 13

3 Discretization, scaling, and solution method 15

3.1 Discretized Fluid Flow Equations . . . . . . . . . . . . 15

3.1.1 Time implicit method . . . . . . . . . . . . . 16

3.1.2 The semi-implicit method . . . . . . . . . . . . 17

3.1 .3 Solution method . . . . . . . . . . . . . . . . 17

3.1.4 Convergence criteria, scaling and preconditioner . . . . . 18

3.1 .5 Numerical flux . . . . . . . . . . . . . . . . . . . . 19

4 Verification 20

4.1 Temporal convergence . . . . . . . . . . . . . . . . 20

4.2 Spatial convergence . . . . . . . . . . . . . . . . 21

4.3 Mesh refinement with an analytical solution . . . . . . . . . 22 
5 Pressurized Water Reactor (PWR) 29

5.1 Characteristic data for PWRs . . . . . . . . . . . . . . . 29

5.2 Steady-state and transient . . . . . . . . . . . . . . . . . 29

$5.2 .1 \quad$ Steady-state . . . . . . . . . . . . . . . . 29

5.2.2 Pseudo-transient to steady-state (with pumps on) . . . . 33

5.2 .3 Model uncertainty . . . . . . . . . . . . . . . . . 35

5.3 Study of a transient: pumps off . . . . . . . . . . . . 37

5.3 .1 Transient: pumps off . . . . . . . . . . 38

5.3.2 Final steady-state after the transient . . . . . . . . . . 40

5.3 .3 Model sensitivity . . . . . . . . . . . . . . . . . . . . 43

5.4 Sensitivity analysis . . . . . . . . . . . . . . . . 44

6 Conclusions and future work 46

$\begin{array}{lll}7 & \text { Appendix } & 47\end{array}$ 


\section{Chapter 1}

\section{Introduction}

The goal of this study is to assess the impact of various flow models for a simplified primary coolant loop of a light water nuclear reactor. The various fluid flow models are based on the Euler equations with an additional friction term, gravity term, momentum source, and energy source. The geometric model is purposefully chosen simple and consists of a one-dimensional (1D) loop system in order to focus the study on the validity of various fluid flow approximations. The 1D loop system is represented by a rectangle; the fluid is heated up along one of the vertical legs and cooled down along the opposite leg. A pressurizer and a pump are included in the horizontal legs. The amount of energy transferred and removed from the system is equal in absolute value along the two vertical legs. The various fluid flow approximations are compressible vs. incompressible, and complete momentum equation vs. Darcy's approximation. The ultimate goal is to compute the fluid flow models' uncertainties and, if possible, to generate validity ranges for these models when applied to reactor analysis. We also limit this study to single phase flows with low-Mach numbers. As a result, sound waves carry a very small amount of energy in this particular case. A standard finite volume method is used for the spatial discretization of the system. 


\section{Chapter 2}

\section{Geometrical and mathematical models}

The test-bed code developed in this work aims at studying the primary cooling of a nuclear reactor, either a Pressurized Water Reactor, a Sodium Fast Reactor, or a Hight Temperature Gas Reactor. Some simplifying assumptions are made: 1D geometry and single-phase low-Mach flow. Within this model, various fluid

flows approximations are assessed. We present here the geometry utilized, the assumptions made, the various flow approximations, and the numerics employed to solve in space/time the equations obtained.

\section{$2.1 \quad$ Model geometry}

The 1D loop system is represented by a rectangle, shown in Fig. 2.1. The fluid is heated up along the left vertical legs of length $L_{1}$ and cooled down along the opposite leg. The cooling process is to mimic the presence of steam generators. A pressurized is present on the top horizontal length of length $L_{2}$ and a pump is included in the lower horizontal leg. These two components are smeared over a certain portion of the leg length in order to avoid making the pressurizer and the pump action size grid dependent. 


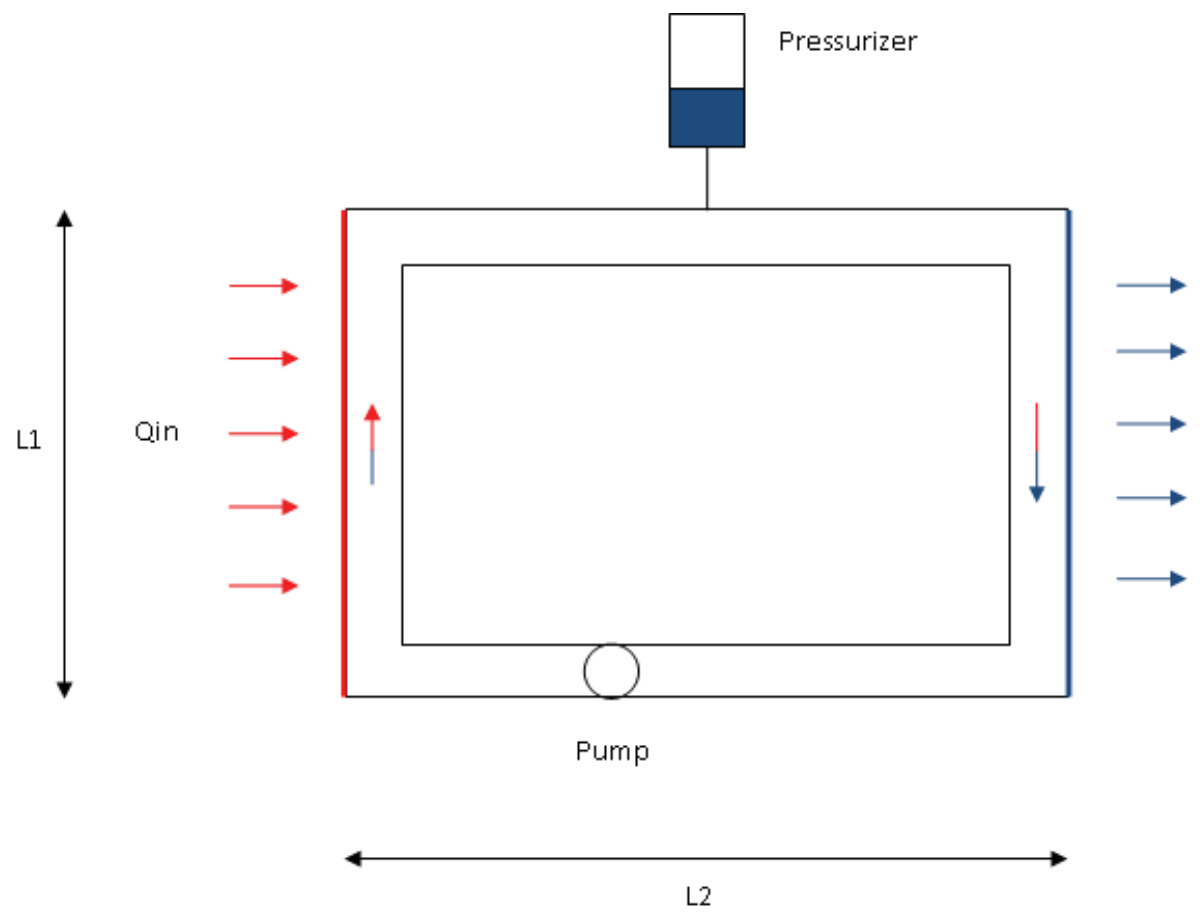

Figure 2.1: Loop geometry.

\subsection{Assumptions for the model}

The following assumptions are made for this 1D model and are detailed in this section:

- The heat source and sink are expressed in term of the heat transfer coefficient and the fluid temperature as follows:

$$
Q= \pm h A\left(T_{w}-T_{f}\right),
$$

where $h$ is the heat transfer coefficient, $A$ is the exchange area between the wall and the fluid, and, $T_{f}$ and $T_{w}$ are the fluid and wall temperature, respectively. In the left vertical leg, this source term is positive (heat is applied) and in the opposite leg, it is negative (heat sink). Heat and sink source terms are distributed over the entire length $L_{1}$ of these two legs.

- The heat transfer coefficient $h$ is constant and is taken to be the same for forced convection and natural convection processes. In practice, two different correlations should be employed since the physics are different in these two cases (this can be modified in a subsequent version of this 
test-bed code). $T_{w}$ is a given constant equal to $T_{w c o r e}$ in the heated leg, and $T_{w s g}$ in the cooled leg, where $T_{w c o r e}$ and $T_{w s g}$ are reactor-dependent.

- The heat capacity $C_{v}$ is temperature- and pressure-independent. This is a good assumption for some fluids, such as water, for a range of temperature and pressure. For other coolants, such as gas, this approximation is not representative of the real behavior of the fluid.

- The sound speed, $c$, is supposed to be constant even if it usually depends on the temperature and the pressure. This is a good approximation for liquids but not for gases.

- The Equation Of State (EOS) is linear in temperature and pressure as follows:

$$
\rho=\rho_{0}+\left.\frac{\partial \rho}{\partial P}\right|_{0}\left(P-P_{0}\right)+\left.\frac{\partial \rho}{\partial T}\right|_{0}\left(T-T_{0}\right),
$$

where $\rho, T$ and $P$ are the density, the temperature and the pressure of the fluid, respectively, and $\rho_{0}, T_{0}$ and $P_{0}$ are the fluid proprieties at a particular reference point (the linearization point). $\frac{\partial \rho}{\partial P}$ and $\frac{\partial \rho}{\partial T}$ are defined as the variation of the density relative to the pressure and the temperature, respectively.

- $\frac{\partial \rho}{\partial T}$ is the dilatation of the density due to the temperature. This parameter is assumed constant in this model.

- $\frac{\partial \rho}{\partial P}$ is the dilatation of the density due to the pressure. This parameter is also assumed constant but is different for different sound speeds. Its expression is as follows:

$$
\frac{\partial \rho}{\partial P}=\frac{1}{c^{2}}
$$

- Friction forces are taken account into this model. The friction parameter $f_{w}$ is a function of the Reynolds number and depend on the laminar vs turbulent nature of the flow. Its expression is given in a subsequent section.

Different flow models are implemented:

1. compressible flow,

2. incompressible flow with Boussinesq correction,

3. Darcy's law in either compressible or incompressible settings.

A solution algorithm (and thus a single code) is written, where binary $(0 / 1)$ parameter values are changed in the discretized equations themselves to turn on or off any of these three flow models seamlessly. The following subsections give the equations used and the different approximations related to each model. 


\subsubsection{Compressible and incompressible flows}

A flow is defined as compressible when its density can change with respect to pressure. In general, this is the case when the Mach number of the flow exceeds 0.3. To account of the pressure effect, a linearized Equation Of State (EOS) is used and defined as follows:

$$
\rho(P, T)=\rho_{0}\left(P_{0}, T_{0}\right)+\left.\frac{\partial \rho}{\partial P}\right|_{0}\left(P-P_{0}\right)+\left.\frac{\partial \rho}{\partial T}\right|_{0}\left(T-T_{0}\right),
$$

where $\rho(P, T)$ is the density function of the pressure $P$ and temperature $T, \frac{\partial \rho}{\partial P}$ is defined as the inverse of the sound speed square in the fluid (see Eq. (2.3)), $P_{0}$ and $T_{0}$ are the pressure and temperature at the point of linearization respectively and $\frac{\partial \rho}{\partial T}$ is the change in density due to the temperature. In this study,

the parameter $\frac{\partial \rho}{\partial P}$ is held constant for a given fluid. This EOS is derived from a Taylor series development at the point of pressure $P_{0}$ and is a good approximation since there is no change of phase in our model.

In the case of incompressible flows, the term $\frac{\partial \rho}{\partial P}$ is equal to zero, which is equivalent to set the sound speed to infinity. In other terms, the sound waves travel through the entire domain with an infinite velocity and the density is constant and equals to $\rho_{0}$.

It has to be noted that the change in temperature can lead to changes in the density. The Boussinesq correction accounts for this effect and is described in the following section.

\subsubsection{Boussinesq correction}

For non-isothermal fluids, the density can vary with temperature. The Boussinesq correction can be employed to allow the density to be temperature-dependent. This assumption is necessary to account for buoyancy forces.

$$
\rho(T)=\rho_{0}\left(T_{0}\right)+\left.\frac{\partial \rho}{\partial T}\right|_{0}\left(T-T_{0}\right),
$$

where $\rho(T)$ is a temperature-dependent density function, $\rho_{0}$ is the density value at the temperature $T_{0}, \frac{\partial \rho}{\partial T}$ is the changes in density due to the temperature (this value is generally negative), and $T_{0}$ is the temperature value at the linearization point. Variations of density with respect to temperature are very important because they give rise to buoyancy forces and, thus, to natural convection.

In the case of compressible flow, the EOS is modified as follows:

$$
\rho(P, T)=\rho_{0}\left(P_{0}, T_{0}\right)+\left.\frac{\partial \rho}{\partial P}\right|_{0}\left(P-P_{0}\right)+\left.\frac{\partial \rho}{\partial T}\right|_{0}\left(T-T_{0}\right) .
$$


Compressible flows will always be treated employing Eq. (2.6) and, hence, density is both pressure- and temperature-dependent.

For incompressible flows, the pressure-dependent term of the EOS is removed and thus the density is only temperature-dependent. Two incompressible cases are distinguished according to their Mach number:

1. In the case of low Mach number flow, the density can only be temperaturedependent in the buoyancy term of the momentum equation (denoted by the use of $\hat{\rho}$ in later sections) and is held constant in all other terms of the mass, momentum, and energy equations. The essence of Boussinesq correction is that gravity is sufficiently strong to make the fluid specific weight appreciably different between two fluid positions. In this case, the acceleration term $\frac{D v}{D t}$ is small when compared to the gravity term. This case is the purpose of this study.

2. In the case of high Mach number flows, the density has to be computed with the EOS in all terms of the momentum equation. The acceleration term can be large enough to compensate for the gravity effects.

In the case of our study (low Mach number), the Boussinesq correction is used in order to account for buoyancy forces.

\subsection{Fluid flow equations with wall friction, grav- ity, pump and external energy source terms}

Modified Euler Equations, coupled with an equation of state relation, are employed to solve the 1D loop system described above. The Euler equations are used as a starting point and are modified in the sense that (1) the effect of viscosity has been added and modeled as wall friction, and, (2) gravity is accounted for. Friction forces and gravity forces are the only external forces considered. An energy source term, denoted by $Q$, is also present in the energy equation to model the heat source/sink due to the nuclear core/steam generator, respectively. In order to account for the pump in the model, a momentum source term is added. With these modifications, the fluid equations are:

$$
\begin{aligned}
\partial_{t} \rho+\partial_{x}(\rho u) & =0 \\
\partial_{t}(\rho u)+\partial_{x}\left(\rho u^{2}\right)+\partial_{x} P+\rho \vec{g} \cdot \overrightarrow{u_{x}}+f_{w} \frac{\rho}{D_{h}}|u| u & =F_{\text {pump }}(x, t) \\
\partial_{t}\left(\rho e+\frac{\rho u^{2}}{2}\right)+\partial_{x}\left(\left(\rho e+\frac{\rho u^{2}}{2}\right) u\right)+\partial_{x}(u P) & =Q(x, t)+\mathcal{P}_{g}+\mathcal{P}_{f}+\mathcal{P}_{\text {pump }}
\end{aligned}
$$

The Equation Of State is:

$$
\rho=\rho_{0}+\left.\frac{\partial \rho}{\partial P}\right|_{0}\left(P-P_{0}\right)+\left.\frac{\partial \rho}{\partial T}\right|_{0}\left(T-T_{0}\right)
$$


In the above equations, $\rho$ is the fluid density, $t$ the time variable, $x$ the spatial coordinate, $u$ the fluid velocity, $P$ the pressure, $g$ the gravity, $F_{\text {pump }}$ the pump force, $e$ the internal energy, $D_{h}$ the hydraulic diameter, and, $\rho_{0}, P_{0}$ and $e_{0}$ the fluid density, pressure and internal energy, respectively, at a given pressure and temperature. $\mathcal{P}_{g}, \mathcal{P}_{\text {pump }}$ and $\mathcal{P}_{f}$ are the powers of the gravity, pump and friction forces, respectively. The gravity is taken to be in opposite direction of the velocity vector in the left leg of the loop. The energy source term $Q$ is given by Eq. (2.1) in which the wall temperature and the heat transfer are constant. There is not mass source in the continuity equation since it is a closed loop (this assumption is not correct for the cells linked to the pressurizer). All variables depend on space and time.

These equations correspond to the conservative form of Euler Equations with a non-zero right-hand-side and can be written in the general form as follows:

$$
\partial_{t} U+\partial_{x} F(U)=S
$$

where $S$ is a source vector that will be described later. $U$ and $F(U)$ are as follows:

$$
U=\left[\begin{array}{c}
\rho \\
\rho u \\
\rho e+\frac{1}{2} \rho u^{2}
\end{array}\right] \text { and } F(U)=\left[\begin{array}{c}
\rho u \\
\rho u^{2}+P \\
u\left(\rho e+\frac{1}{2} \rho u^{2}\right)+u P
\end{array}\right]
$$

$U$ is the vector of conservative variables: density, momentum, and total energy. The source term is:

$$
S=\left[\begin{array}{c}
0 \\
-\rho \vec{g} \cdot \overrightarrow{u_{x}}-f_{w} \frac{\rho}{D_{h}}|u| u+F_{\text {pump }}(x, t) \\
Q(x, t)+\mathcal{P}_{g}+\mathcal{P}_{f}+\mathcal{P}_{\text {pump }}
\end{array}\right]
$$

\subsubsection{Primitive variables}

Usually, one solves the Euler Equations in term of conservative variables: density, momentum, and total energy. In reactor safety codes, the solution variables are typically density, velocity and temperature. Therefore, we need to convert the above system of equations in terms of these primitive variables. This will yield the so-called non-conservative form of the fluid equations. The following paragraph is dedicated to deriving the non-conservative equations expressed in terms of the primitive variables.

\subsubsection{The continuity equation and momentum equation}

The continuity equation is unchanged :

$$
\partial_{t} \rho+\partial_{x}(\rho u)=0 .
$$

The momentum equation is modified as follows. The partial derivatives are expanded for each variable. The assumption required is that the functions 
are smooth. In the following, all forces (pressure, gravity and friction forces) appearing in the momentum equation are denoted by $F$ for conciseness. Using the product rule of differentiation the momentum equation yields:

$$
\begin{aligned}
\partial_{t}(\rho u)+\partial_{x}\left(\rho u^{2}\right) & =\rho \partial_{t} u+u \partial_{t} \rho+\rho u \partial_{x} u+u \partial_{x}(\rho u) \\
& =\rho \partial_{t} u+\rho u \partial_{x} u+u\left(\partial_{t} \rho+\partial_{x}(\rho u)\right) \\
& =F+F_{\text {pump }},
\end{aligned}
$$

where $F_{\text {pump }}$ is the pump force. By using the continuity equation, Eq. (2.15) can be further simplified:

$$
\rho \partial_{t} u+\rho u \partial_{x} u=F+F_{\text {pump }}
$$

This is the non-conservative form of the momentum equation. The non-conservative denomination is due to the fact that (1) a flux value can no longer be defined as in the case of the conservative form and (2) the velocity is not a conservative variable.

\subsubsection{The energy equation}

Let us recall the energy equation in its conservative form:

$$
\partial_{t}(\rho E)+\partial_{x}(\rho E u)+\partial_{x}(u p)=Q(x, t)+\mathcal{P}_{g}+\mathcal{P}_{f}+\mathcal{P}_{\text {pump }},
$$

where $E, \rho, u$ are the total energy per unit mass and volume, the density and the velocity respectively. $Q(x, t)$ is the heat source or sink in the system. $\mathcal{P}_{g}$, $\mathcal{P}_{\text {pump }}$ and $\mathcal{P}_{f}$ are the gravity, pump and friction powers. As before, we expand the partial derivatives using the product rule for differentiation:

$$
\begin{aligned}
\rho \partial_{t} E & +E \partial_{t} \rho+\rho u \partial_{x} E+E \partial_{x}(\rho u)+\partial_{x}(u p) \\
& =E\left(\partial_{t} \rho+\partial_{x}(\rho u)\right)+\rho \partial_{t} E+\rho u \partial_{x} E \\
& =Q(x, t)+\mathcal{P}_{g}+\mathcal{P}_{f}+\mathcal{P}_{\text {pump }} .
\end{aligned}
$$

Employing the continuity equation to simplify the equation above yields:

$$
\rho \partial_{t} E+\rho u \partial_{x} E=Q(x, t)+\mathcal{P}_{g}+\mathcal{P}_{f}+\mathcal{P}_{\text {pump }} .
$$

The last step to obtain the non-conservative form of the equation consists in replacing the total energy $E$ by the internal and kinetic energies, $e$ and $\frac{1}{2} u^{2}$,

$$
E=e+\frac{1}{2} u^{2}
$$

Since we are interested in solving the energy equation for temperature, the internal energy $e$ is expressed as a function of the temperature $T$ and the heat capacity $C_{v}$ as follows:

$$
e=C_{v} T
$$


The heat capacity $C_{v}$ is constant in this model as stated in the model assumptions. Then, Eq. (2.19) yields:

$$
\begin{aligned}
& \rho C_{v} \partial_{t} T+\rho \partial_{t} \frac{u^{2}}{2}+\rho u C_{v} \partial_{x} T+\rho u \partial_{x} \frac{u^{2}}{2}+u \partial_{x} P+P \partial_{x} u \\
& \quad=\rho C_{v} \partial_{t} T+\rho u C_{v} \partial_{x} T+P \partial_{x} u+u\left(\rho \partial_{t} u+\rho u \partial_{x} u+\partial_{x} P\right) \\
& \quad=Q(x, t)+\mathcal{P}_{g}+\mathcal{P}_{f}+\mathcal{P}_{\text {pump }}
\end{aligned}
$$

Finally, using the non-conservative momentum expression of Eq. (2.16), Eq. (2.22) becomes:

$\rho C_{v} \partial_{t} T+\rho u C_{v} \partial_{x} T+P \partial_{x} u+u\left(F_{g}+F_{f}+F_{\text {pump }}\right)=Q(x, t)+\mathcal{P}_{g}+\mathcal{P}_{f}+\mathcal{P}_{\text {pump }}$,

where $F_{g}, F_{\text {pump }}$ and $F_{f}$ are the gravity, pump and the friction forces. To further simplify this equation, recall the definition of the power: a force multiply by the velocity. As a result, in the left-hand-side of the equation, the friction, pump and gravity power are recognized, so that the non-conservative form of the energy equation is:

$$
\rho C_{v} \partial_{t} T+\rho u C_{v} \partial_{x} T+P \partial_{x} u=Q
$$

Using the non-conservative form of fluid flow equations along with the EOS, the system can be solved in term of primitive variables.

\subsection{Pump and pressurizer models}

\subsubsection{Pump model}

The pump is implemented as a gravity force since it introduces artificial gravity in the system.

$$
F_{\text {pump }}=\rho g s_{p},
$$

where $F_{\text {pump }}$ is the pump force in the momentum equation, $\rho$ and $g$ are the density and the gravity acceleration, respectively, and $s_{p}$ is the pump strength. The value of $s_{p}$ is selected in order to obtain a fluid velocity representative of the reactor systems modeled. The pump is uniformly distributed over a given length (user-defined input value) so that the pump is independent of the grid size. A ramp can also be set in the code in order to study pump transients (pump coast down).

\subsubsection{Pressurizer model}

The purpose of the pressurizer is to set the pressure at a given point in the system. In our simplified model, we are not interested in keeping track of the fluid level in the pressurizer. However, some fluid is exchanged between the loop and the pressurizer in order to maintain the pressure below a given value set by the operator. The pressurizer temperature is set equal to the temperature 
of the cell linked to it. Since the fluid can flow in and out of the loop into the pressurizer, a pressurizer velocity $u_{p}$ has to be computed. As a result, a momentum equation is added to the residual vector for each cell linked to the pressurizer and given in Eq. (2.26).

$$
\rho_{p} \partial_{t} u_{p}+\rho_{p} u_{p} \partial_{x} u_{p}=F,
$$

where $u_{p}$ is the pressurizer velocity and $\rho_{p}$ is the pressurizer density (this is the only equation solved for the pressurizer; $\rho_{p}$ is determined from the EOS knowing (i) the temperature of the cells linked to the pressurizer and (ii) the pressurizer pressure).

In addition, the pressurizer behaves either as a heat sink or source so that its presence requires an additional term in the continuity and energy equations in the cells concerned (a mass and energy source or sink respectively):

$$
\begin{aligned}
m_{\text {source }} & =\frac{\left(\widetilde{\rho u_{p}}\right)}{\Delta x} \\
e_{\text {source }} & =C_{v} \frac{\left(\widetilde{\rho T u_{p}}\right)}{\Delta x}
\end{aligned}
$$

where $u_{p}, T, \rho$ are the velocity, the temperature and the density, respectively, $C_{v}$ is the heat capacity of the fluid and $\Delta x$ is the grid size. $\left(\widetilde{\rho u_{p}}\right)$ and $(\widetilde{\rho T u})$ are numerical fluxes obtained by applying a simple upwind scheme. The numerical scheme is further discussed in Section 3.1.5. As in the case of the pump, the pressurizer model is independent of the space grid size in order to avoid having strong pressure discontinuities in the system.

\subsection{Fluid flow models}

\subsubsection{Laminar and turbulent flows}

Laminar and turbulent flows correlations are utilized for the friction term, depending on the Reynolds number. The correlations presented here are used for transient and steady-state runs. In the case of laminar flows, the friction factor $f_{w}$ is a function of the Reynold number $R e$ as follows:

$$
f_{w}=\frac{64}{R e}
$$

Th expression Eq. (2.29) is known as the Darcy-Weisbach factor [1]. For turbulent flows, the Blasius correlation is used and given below:

$$
f_{w}=0.079 R e^{-0.25}
$$

The Blasius correlation can be used under some particular conditions: it is applicable well for liquid water but requires either some modifications or the 
use of another correlation in the case of gases. Since only PWRs are studied here, only the Blasius correlation is given.

The Reynold number is a function of velocity $u$, hydraulic diameter $D_{h}$, viscosity $\mu$, and density $\rho$, and is given as follows:

$$
R e=\frac{\rho D_{h}|u|}{\mu}
$$

\subsubsection{Darcy's law}

Darcy's law can be used to model flows in porous media. For reactor applications, this could be, for instance, fluid flow a pebble bed gas reactor. Darcy's law assumes a porous medium and a laminar flow and is obtained from the momentum equation by setting the time dependent term and the convection term set to zero (for both compressible and incompressible flows).

$$
\rho g+f_{w} \frac{\rho}{D_{h}} u|u|+\partial_{x} P=0
$$

Assuming a laminar flow, the friction force $F_{f}$ appearing the momentum equation can be expressed as follows:

$$
\begin{aligned}
F_{f} & =f_{w} \frac{\rho}{D_{h}} u|u| \\
& =\frac{64}{\operatorname{Re}} \frac{\rho}{D_{h}} u|u| \\
& =\frac{64}{D_{h}^{2}} \mu u
\end{aligned}
$$

where Eq. (2.29) was employed. Hence, the velocity can be given by a linear equation:

$$
u=-\left(\frac{\partial P}{\partial x}+\rho g\right) \frac{D_{h}^{2}}{64 \mu}
$$

\subsection{Final forms of the equations}

The fluid flow models (compressible, incompressible, with or without Boussinesq correction, with or without Darcy's law approximation) can be written in a generic form in which each model can be turned on and off using some binary $\alpha$ parameters. Hence, a single code and solution algorithm is employed to assess in a consistent fashion the effects of the various models. The generic form of the equations with the $\alpha$ parameters are given: 


$$
\begin{aligned}
& \partial_{t} \rho+\partial_{x}(\rho u)=0 \\
& \alpha_{4}\left(\rho \partial_{t} u+\rho u \partial_{x} u\right)+\hat{\rho} g\left(1-s_{p}\right)+f_{w} \frac{\rho}{D_{h}}|u| u+\partial_{x} P=0 \\
& \rho C_{v} \partial_{t} T+\rho u C_{v} \partial_{x} T+P \partial_{x} u=Q \\
& \rho=\rho_{0}+\left.\alpha_{1} \frac{\partial \rho}{\partial P}\right|_{0}\left(P-P_{0}\right)+\alpha_{2}\left.\frac{\partial \rho}{\partial T}\right|_{0}\left(T-T_{0}\right) \\
& \hat{\rho}=\rho_{0}+\left.\alpha_{1} \frac{\partial \rho}{\partial P}\right|_{0}\left(P-P_{0}\right)+\left.\alpha_{3} \frac{\partial \rho}{\partial T}\right|_{0}\left(T-T_{0}\right)
\end{aligned}
$$

The different combinations of $\alpha_{i}$ are summarized in Table 2.1.

Table 2.1: Alpha combinations

\begin{tabular}{|l||c|c|c|c|}
\hline Flow option & $\alpha_{1}$ & $\alpha_{2}$ & $\alpha_{3}$ & $\alpha_{4}$ \\
\hline \hline Compressible & 1 & 1 & 1 & 1 \\
\hline Incompressible without Boussinesq & 0 & 0 & 0 & 1 \\
\hline Incompressible with Boussinesq & 0 & 0 & 1 & 1 \\
\hline Compressible Darcy & 1 & 1 & 1 & 0 \\
\hline Incompressible Darcy with Boussinesq & 0 & 0 & 1 & 0 \\
\hline
\end{tabular}

Note that the incompressible flow model without the Boussinesq correction is not employed here (natural convection cannot occur under this assumption). Only two cases are presented in this report: compressible and incompressible flow model with Boussinesq correction. Darcy's law will be used in future work, e.g., for High Temperature Gas Reactors. 


\section{Chapter 3}

\section{Discretization, scaling, and solution method}

This section aims at describing the numerical method used in the pilot code. After giving the implicit and explicit forms of the Euler equations, the Jacobianfree-Newton-Krylov method (solution method) is explained. The other subsections deal with numerical techniques used such as scaling, preconditioner, convergence criteria and numerical flux.

\subsection{Discretized Fluid Flow Equations}

A standard finite volume (FV) spatial discretization based on staggered grids is employed and recalled below. The stored variables are the temperature, the pressure, and the velocity (density depends on the temperature and the pressure through the EOS so that it is not required to store it). In a staggered grid technique, two grids are employed; there is an offset of half a cell in between the grids so that the edge of cells of the first grid corresponds to the middle point of cell of the second grid. This method makes the FV stable and numerical fluxes can be easily computed for each equation. Only the continuity, momentum, and energy equations are discretized using the grids shown in Fig. 3.1. The EOS does not need to be discretized. Two temporal discretizations are employed, the implicit and semi-implicit methods. These schemes are described next. 


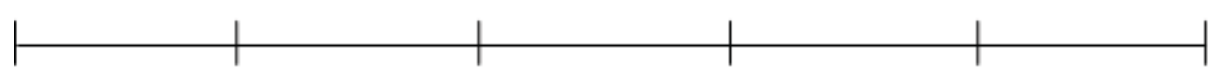

Grid for the temperature, the density and the pressure.

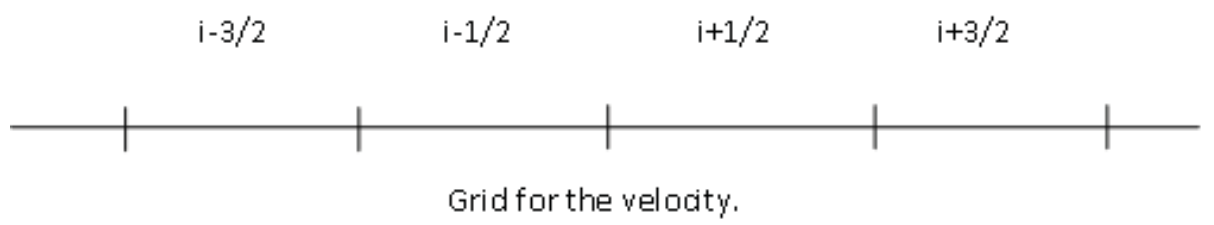

Figure 3.1: Staggered grids for the FV spatial discretization. Top: grid for $T$, $\rho$, and $P$; Bottom: grid for $u$

\subsubsection{Time implicit method}

Backward (implicit) Euler time integration yields the following discretized equations.

Integrating the continuity expression, Eq. (2.14), over spatial cell $i$ yields:

$$
\frac{\rho_{i}^{n+1}-\rho_{i}^{n}}{\Delta t}+\frac{(\widetilde{\rho} u)_{i+\frac{1}{2}}^{n+1}-(\widetilde{\rho} u)_{i-\frac{1}{2}}^{n+1}}{\Delta x}=0
$$

Integrating the momentum expression, Eq. (2.16), over spatial cell $i+\frac{1}{2}$ yields:

$$
\begin{aligned}
\bar{\rho}_{i+\frac{1}{2}}^{n+1} \frac{u_{i+\frac{1}{2}}^{n+1}-u_{i+\frac{1}{2}}^{n}}{\Delta t}+ & \bar{\rho}_{i+\frac{1}{2}}^{n+1} u_{i+\frac{1}{2}}^{n+1} \frac{\widetilde{u}_{i+1}^{n+1}-\widetilde{u}_{i}^{n+1}}{\Delta x} \\
& +\frac{P_{i+1}^{n+1}-P_{i}^{n+1}}{\Delta x}+\overline{\hat{\rho}}_{i+\frac{1}{2}}^{n+1} g=F_{p u m p, i+\frac{1}{2}}^{n+1}
\end{aligned}
$$

Integrating the energy expression, Eq. (2.24), over spatial cell $i$ yields:

$$
\begin{aligned}
C_{v} \frac{(\rho T)_{i}^{n+1}-(\rho T)_{i}^{n}}{\Delta t}+C_{v} \frac{(\widetilde{\rho T} u)_{i+\frac{1}{2}}^{n+1}-(\widetilde{\rho T} u)_{i-\frac{1}{2}}^{n+1}}{\Delta x} & \frac{\widetilde{u}_{i+\frac{1}{2}}^{n+1}-\widetilde{u}_{i-\frac{1}{2}}^{n+1}}{\Delta x}=Q_{i}^{n+1}
\end{aligned}
$$


where $\Delta t$ and $\Delta x$ are the temporal and spatial discretization grid sizes, $n$ and $i$ are temporal and spatial indices, respectively. The variable $\hat{\rho}$ can be different from the density variable $\rho$ when the Boussinesq correction is applied: for compressible fluids, the two variables are identical; for incompressible with the Boussinesq correction, $\hat{\rho}$ depends on temperature through the EOS, whereas $\rho$ is constant. The notation $\bar{\rho}_{i+\frac{1}{2}}^{n+1}$ denotes as the average density on cells $i$ and $i+1$ at time $n+1$ :

$$
\bar{\rho}_{i+\frac{1}{2}}^{n+1}=\frac{\rho_{i+1}^{n+1}+\rho_{i}^{n+1}}{2}
$$

The notation $(\widetilde{\rho} u)_{i+\frac{1}{2}}^{n+1}$ denotes the numerical flux of $\rho u$ on edge $i+\frac{1}{2}$ of cell $i$ at time $n+1$ and is computed using the upwind scheme (see section 3.1.5).

\subsubsection{The semi-implicit method}

In the semi-implicit method, some variables are taken explicit and some others implicit. In doing so, the number of Newton iterations can be reduced and the code runs faster. The equations are given:

$$
\begin{gathered}
\frac{\rho_{i}^{n+1}-\rho_{i}^{n}}{\Delta t}+\frac{(\widetilde{\rho} u)_{i+\frac{1}{2}}^{n}-(\widetilde{\rho} u)_{i-\frac{1}{2}}^{n}}{\Delta x}=0 \\
\bar{\rho}_{i+\frac{1}{2}}^{n} \frac{u_{i+\frac{1}{2}}^{n+1}-u_{i+\frac{1}{2}}^{n}}{\Delta x}+\bar{\rho}_{i+\frac{1}{2}}^{n} u_{i+\frac{1}{2}}^{n} \frac{\widetilde{u}_{i}^{n}-\widetilde{u}_{i}^{n}}{\Delta x}+\frac{P_{i+1}^{n+1}-P_{i}^{n+1}}{\Delta x}+\overline{\hat{\rho}}_{i+\frac{1}{2}}^{n} g=0 \\
C_{v} T_{i}^{n+1} \frac{\rho_{i}^{n+1}-\rho_{i}^{n}}{\Delta t}+C_{v} \rho_{i}^{n+1} \frac{T_{i}^{n+1}-T_{i}^{n}}{\Delta t} \\
+C_{v} \frac{(\widetilde{\rho T} u)_{i+\frac{1}{2}}^{n+1}-(\widetilde{\rho T} u)_{i-\frac{1}{2}}^{n+1}}{\Delta x}+P_{i}^{n} \frac{u_{1+\frac{1}{2}}^{n+1}-u_{1-\frac{1}{2}}^{n+1}}{\Delta x}=0
\end{gathered}
$$

In the semi-implicit formulation, some variables are treated explicitly. In the continuity equation, the mass flow is now an explicit variable. The velocity is taken at time $n$. In the momentum equation, all terms are explicit but the pressure which is implicit. In the energy equation, the velocity is implicit in all terms of the equations and only the pressure is explicit. The time dependent term is broken down into two terms.

\subsubsection{Solution method}

After temporal and spatial discretization, the above problem forms a set of nonlinear equations for the unknowns at time $t^{n+1}$. There are $N=3 \times n_{e l}$ unknowns: 3 types of variables per cell $(\rho, u, T) ; 1$ unknown per variable per cell (FV method); and a total of $n_{e l}$ cells. The nonlinear system of equation can be expressed as follows

$$
\mathcal{F}\left(U^{n+1}\right)=0
$$


(we will drop the ${ }^{n+1}$ superscript in the rest of this section). $\mathcal{F}$ is a nonlinear vector of $N$ components. This nonlinear system is solved iteratively (iteration index is $\ell$ ) using Newton's method:

$$
\begin{aligned}
\left.\frac{\partial \mathcal{F}}{\partial U}\right|_{U^{\ell}} \delta U & =-\mathcal{F}\left(U^{\ell}\right) \\
U^{\ell+1} & =U^{\ell}+\delta U
\end{aligned}
$$

until $\left|\mathcal{F}\left(U^{\ell}\right)\right|<\epsilon$. The Jacobian matrix $J=\frac{\partial \mathcal{F}}{\partial U}$ is never formed. Rather, the (non-symmetric) linear system $J \delta U=-\mathcal{F}$ is solved using a Krylov method (GMRES). In Krylov methods, only the action of the matrix on a Krylov vector $\nu$ is required. Here, this is approximated using a finite difference formula as follows

$$
J \nu \simeq \frac{\mathcal{F}\left(U^{\ell}+\varepsilon \nu\right)-\mathcal{F}\left(U^{\ell}\right)}{\varepsilon}
$$

where $\varepsilon$ is typically the square-root of machine precision. Hence, only a function that evaluates the nonlinear residual $\mathcal{F}$ is required. This forms the basics of the Jacobian-free Newton-Krylov (JFNK) techniques.

\subsubsection{Convergence criteria, scaling and preconditioner}

Matlab is used to code the modified Euler Equations. The code is broken down in many functions in order to make it easy to change. It has an input file that allows changing all the parameters relative to the fluid (viscosity, heat capacity, $\ldots)$ and the system $\left(Q_{\text {in }}, Q_{\text {out }}, \ldots\right)$. Some further details about the code are given:

- The variables (pressure and temperature) are scaled in order to reduce the condition number of the Jacobian matrix. This should avoid the code to breakdown at very low Mach number which might be the case as the pump will be turned off [3].The temperature and pressure are scaled as follows:

$$
\begin{array}{r}
P(x, t)=P_{0}+c P^{\prime}(x, t) \\
T(x, t)=d T^{\prime}(x, t),
\end{array}
$$

where $P(x, t)$ and $P_{0}$ are the pressure in the loop and the average pressure in the loop respectively. $P^{\prime}(x, t)$ is the variation of the pressure around the average pressure of the system. $c$ is a constant that can be adjusted in order to make $P^{\prime}(x, t)$ smaller or bigger. The variables $T(x, t)$ and $T^{\prime}(x, t)$ are the temperatures of the fluid. $d$ is also a constant that can be adjusted. The process of scaling aims at making all variables between 1 and 10 in the solution vector by making the condition number smaller. Scaling reduced the condition number from $10^{16}$ to $10^{8}$. It was tested that the numerical solution is not scaling-dependent. 
- Two convergence criteria are used for the GMRES method. The exact Newton is used (i.e., the nonlinear system is solved to a given tolerance) and an additional criterion, given in Eq. (3.14), is set in order to force the system to converge when steady state is reached:

$$
\frac{\delta V}{V}<t o l,
$$

where $\delta V$ is the vector computed by the GMRES method, $V$ is the solution vector and $t o l$ is the tolerance $\left(t o l=10^{-8}\right)$.

- The semi-explicit Jacobian matrix (Jacobian matrix of the previous time step is used as a preconditioner in the GMRES method. Computing the Jacobian matrix, however, can be time consuming as a large number of cells is utilized.

- The steady state is detected by computing the heat removed from and supplied to the system. When they equal each other within a given tolerance, it is considered that the steady state has been reached:

$$
Q_{\text {in }}=-Q_{\text {out }}
$$

\subsubsection{Numerical flux}

The numerical flux used here is computed using the upwind method [2]. Let consider three cells $i-1, i$ and $i+1$ and the continuity equation discretized on cell $i$. Two cases are possible at edge $i+\frac{1}{2}$ (these cases are explained with the density but can be applied to any function):

- if the velocity is positive at the given edge, the density $\widetilde{\rho}_{i+\frac{1}{2}}$ equals the density in cell $i$,

- if the velocity is negative at the given edge, the density $\widetilde{\rho}_{i+\frac{1}{2}}$ equals the density in cell $i+1$.

That is

$$
\widetilde{\rho}_{i+\frac{1}{2}}=\left\{\begin{array}{cl}
\rho_{i} & \text { if } u_{i+\frac{1}{2}}>0 \\
\rho_{i+1} & \text { if } u_{i+\frac{1}{2}}<0
\end{array} \quad \forall i \in\left[1, n_{e l}\right]\right.
$$

Similar definitions of numerical fluxes hold for other variables, as required in the spatial FV scheme. This numerical flux technique is called upwind since it accounts for the direction of the fluid in the system. This numerical flux does not conserve the momentum (that is momentum fluxed out of cell $i+\frac{1}{2}$ at face $i$ is not the momentum fluxed into cell $i-\frac{1}{2}$ ) but is stable in all cases. Some other numerical fluxes could be used in future work (e.g., central difference). 


\section{Chapter 4}

\section{Verification}

This chapter aims at studying the temporal and spatial convergence rates. In the case of the incompressible model, an analytical solution is derived and used to quantify the spatial error. The following pseudo transient is used in this chapter to reach a steady-state: a ramp of 9 seconds is set for the source and sink energy, the error is computed at the time $t=20$ seconds in order to have smooth variations in the solutions.

\subsection{Temporal convergence}

The time integration method used is first order in time. Hence, the temporal error $\epsilon_{\Delta t}$ should be decreased by a factor of two each time the time step size is decreased by a factor two.

$$
\begin{aligned}
\epsilon_{\Delta t} & =C \Delta t^{p} \\
\log (\epsilon) & =\log (C)+p \times \log (\Delta t),
\end{aligned}
$$

where $C$ is a constant depending on the system of equations of the time-stepping scheme, and $p$ is the order of the numerical method (it is one in our case since we use Backward Euler). The second form of the equation is used to plot the log of the error as a function of the log of the time step, yielding the numerical slope $p$. In order to avoid any pollution for the spatial discretization error, a very fine spatial mesh is utilized. To perform the temporal convergence analysis, the following time steps, given in seconds, have been employed: $2,1,0.5,0.25,0.125$, 0.0625 and 0.03125 . The average error is computed using successive numerical solutions as follows:

$$
\epsilon_{\Delta t}=\frac{1}{n_{e l}} \sum_{i=1}^{n_{e l}}\left(U(i, t)_{\Delta t}-U(i, t)_{\frac{\Delta t}{2}}\right),
$$

where $\epsilon_{\Delta t}$ is the average diiference between numerical simulations employing time steps $\Delta t$ and $\frac{\Delta t}{2}, n_{e l}$ is the number of cells, and $U(i, t)$ is the solution vector in cell $i$ at time $t$. 
The temperature error is plotted versus the time step in Fig. 4.1.

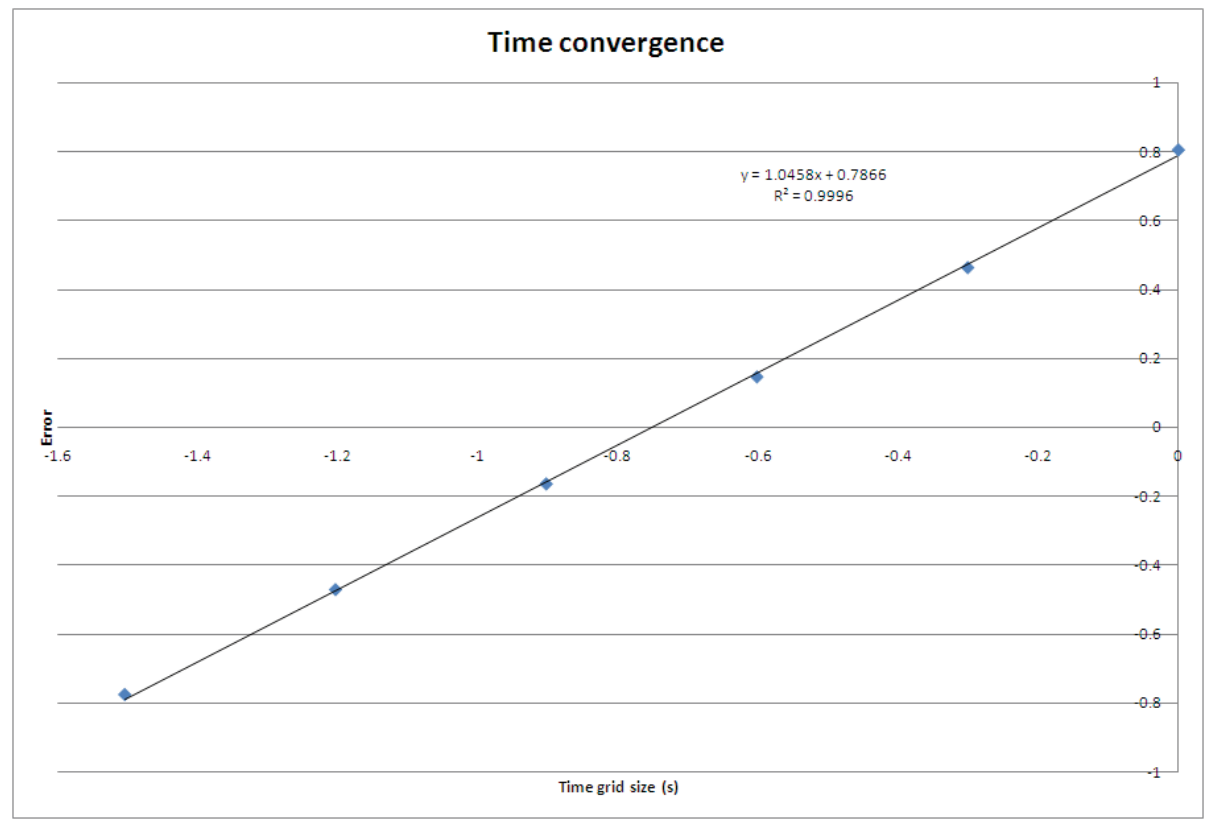

Figure 4.1: Time convergence

The numerically observed slope is 1.0458 , which is close to the expected theoretical value. This proves that the code is indeed first order in time.

\subsection{Spatial convergence}

In this section, the spatial convergence is demonstrated. The method is the following: the space grid size is changed by a factor of two for each new simulation while the time step is constant to a small value to avoid pollution from the temporal discretization error. The error is computed at steady-state with equation Eq. (4.2) and plotted as function of the space grid size in figure Fig. 4.2. A slope of 0.9633 is observed, showing that the code is first order in space. 


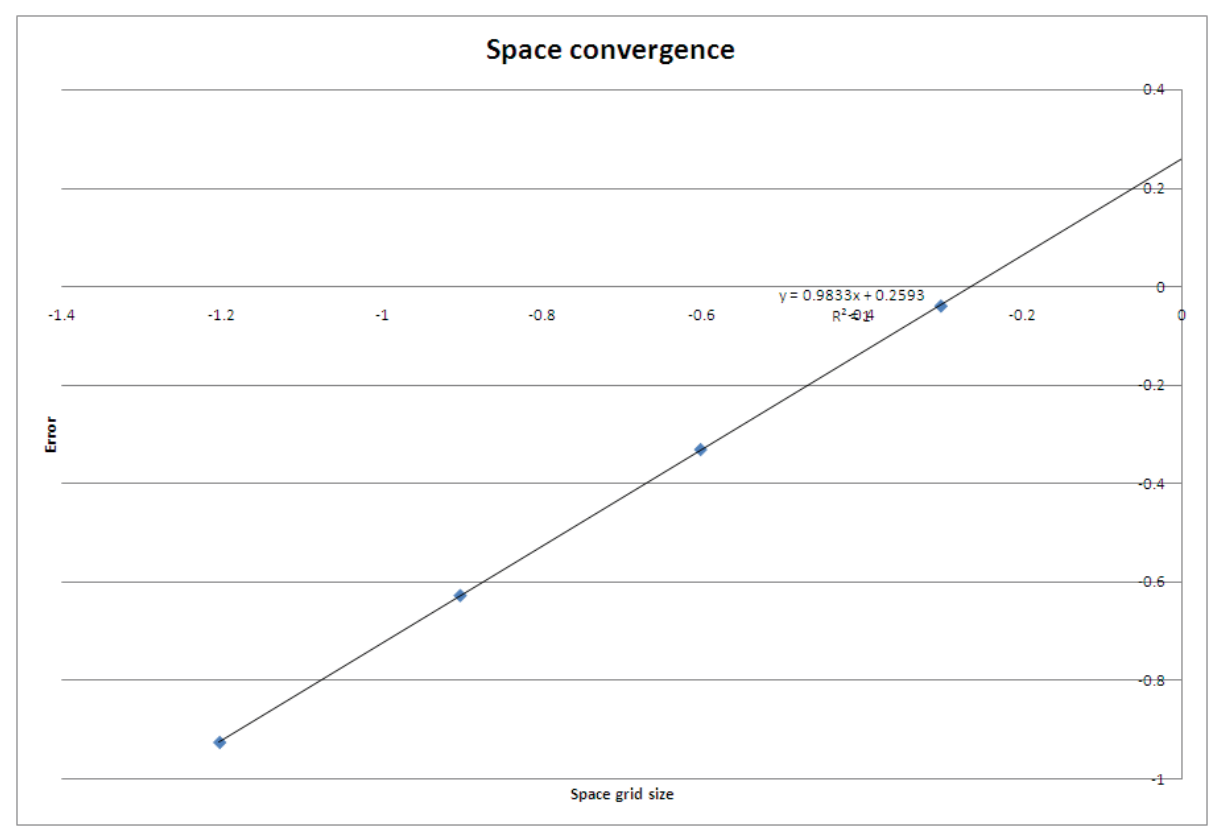

Figure 4.2: Space convergence

\subsection{Mesh refinement with an analytical solution}

In the case of the incompressible model, it is possible to derive an analytical solution from the equations. The modified Euler Equations in the case of the incompressible model (with Boussinesq correction) are recalled:

$$
\begin{aligned}
\partial_{x}(u) & =0 \\
\rho \partial_{t} u+\rho u \partial_{x} u+\partial_{x} P+\hat{\rho} g\left(1-s_{p}\right)+f_{w} \frac{\rho}{D_{h}}|u| u & =0 \\
\rho C_{v} \partial_{t} T+\rho u C_{v} \partial_{x} T+P \partial_{x} u & =Q(x, t) \\
\rho & =\rho_{0} \\
\hat{\rho} & =\rho_{0}+\frac{\partial \rho}{\partial T}\left(T-T_{0}\right)
\end{aligned}
$$

The momentum and energy equations can be simplified using the continuity equation:

$$
\begin{array}{r}
\rho_{0} \partial_{t} u+\partial_{x} P+\hat{\rho} g\left(1-s_{p}\right)+f_{w} \frac{\rho_{0}}{D_{h}}|u| u=0 \\
\rho_{0} C_{v} \partial_{t} T+\rho_{0} u C_{v} \partial_{x} T=Q(x, t) .
\end{array}
$$

An analytical solution can be derived from the equation above by making the steady state assumption. The steps are detailed for the temperature and pres- 
sure profiles along the core and the results will be given for the other legs of the loop (pressurizer, steam generator and pump) in Appendix 7. Along the core, the heat source is $Q(x)=h_{\text {core }} L_{1}\left(T_{\text {wcore }}-T(x)\right)$ in one dimension where $h$ is the heat transfer coefficient, $L_{1}$ is the length of the core, $T_{w c o r e}$ is the wall temperature along the core and $T(x)$ is the fluid temperature profile along the core (it is assumed that the area is $L_{1} \times 1$. Then, the energy equation yields:

$$
\begin{array}{r}
\rho u C_{v} \partial_{x} T=h_{\text {core }} L_{1}\left(T_{w c o r e}-T(x)\right) \\
T(x)+\frac{\rho u C_{v}}{h_{\text {core }} L_{1}} T(x)=T_{w c o r e}
\end{array}
$$

This is a first order differential equation that can be solved analytically. To simplify the equation, let us define $l_{\text {core }}=\frac{\rho_{0} u C_{v}}{h_{c o r e} L_{1}}$ which is the characteristic length of the energy equation. The parameter $l_{\text {core }}$ depends on the fluid velocity so that it will affect the temperature profile as expected. A solution for this type of differential equation is (the temperature is solved as a function of position $x)$ :

$$
T_{\text {core }}(x)=T_{\text {core }}+B e^{\frac{-x}{l_{\text {core }}}}
$$

where $B$ is a yet-to-be-determined constant. Eq. (4.13) gives the temperature profile along the core. We can now derive the pressure profile along the core using the momentum equation. The temperature and the pressure are coupled through the Boussinesq correction in the momentum equation. The pressure along the core is given by Eq. (4.14)

$$
P(x)=\frac{B g}{l_{\text {core }}}\left(e^{\frac{-x}{l_{\text {core }}}}-e^{\frac{-L_{1}}{l_{\text {core }}}}\right)-\left[\left(\rho_{0}+\frac{\rho}{T}\right) g+f_{w} \frac{\rho}{D_{h}}|u| u\right]\left(L_{1}-x\right)+P_{p},
$$

where $P_{p}$ is the pressure in the pressurizer and is an input in the code. Eq. (4.14) gives the pressure as a function of space at steady-state along the core. This analytical solution will be used as a reference for the numerical solution. Of course, the constant $B$ has to be computed. This requires one to compute the temperature profile along the steam generator. The energy equation to solve for the steam generator is the following:

$$
\rho u C_{v} \partial_{x} T=h_{s g} L_{1}\left(T_{w s g}-T(x)\right) T(x)+\frac{\rho u C_{v}}{h_{s g} L_{1}} T(x)=T_{w s g},
$$

where $T_{w s g}$ is the temperature in the steam generator, $h_{s g}$ is the heat transfer coefficient between the fluid and the steam generator. All other variables are defined as above. We also define $l_{s g}=\frac{\rho u C_{v}}{h_{s g} L_{1}}$ as the space scale of the energy equation along the steam generator. The solution technique is the same as before and the solution is:

$$
T_{s g}(x)=T_{w s g}+D e^{\frac{-x}{l_{s g}}}
$$

where $D$ is a constant to be determined. To obtain the constants $B$ and $D$, two equations are required. The first one is obtained by stating that the temperature 
along the pump leg is constant, since in this model, the pump does not modify the energy equation (this assumption is not true along the pressurizer). As a result:

$$
T_{\text {core }}(x=0)=T_{s g}\left(x=2 L_{1}+L_{2}\right),
$$

where $T_{s g}$ and $T_{\text {core }}$ are the temperature in the steam generator and in the core, respectively, and $L_{1}$ and $L_{2}$ are the loop lengths.

The second equation is obtained by considering that the steady-state is reached when the energy put into and removed from the system equal each other:

$$
Q_{\text {in }}=-Q_{\text {out }}
$$

Using these two relations above, the system can be solved and the constants expressed as a function of the boundary conditions (since the system is periodic, the boundary conditions are defined as the temperature in the core and in the stem generator).

$$
\begin{gathered}
D=\frac{T_{\text {wcore }}-T_{w s g}}{e^{-\frac{\left(2 L_{1}+L_{2}\right)}{l_{s g}}}-\frac{l_{s g} h_{s g}}{l_{\text {core }} h_{\text {core }}} \frac{e^{-\frac{\left(2 L_{1}+L_{2}\right)}{l_{s g}}}-e^{-\frac{\left(L_{1}+L_{2}\right)}{l_{s g}}}}{1-e^{-\frac{L_{1}}{l_{\text {core }}}}}} \\
B=\left(T_{\text {wcore }}-T_{w s g}\right)\left(\begin{array}{c}
\frac{1}{1-\frac{l_{s g} h_{s g}}{l_{\text {core }} h_{\text {core }}}} \frac{1-e^{-\frac{L_{1}}{l_{s g}}}}{1-e^{-\frac{L_{1}}{l_{\text {core }}}}}-1
\end{array}\right)
\end{gathered}
$$

As it can be seen in Eq. (4.19) and Eq. (4.20), the constants $B$ and $D$ are independent of the initial conditions. As a result, the numerical solution is unique and does not depend on the initial conditions, in the case of the incompressible model. Since the constants $B$ and $D$ are known, the pressure equations can be derived:

$$
\begin{aligned}
& P(x)=\frac{\left(T_{\text {wcore }}-T_{w s g}\right) g}{l_{\text {core }}}\left(\frac{1}{1-\frac{l_{\text {sg }} h_{\text {sg }}}{l_{\text {core }} h_{\text {core }}} \frac{1-e^{-\frac{L_{1}}{l_{s g}}}}{1-e^{-\frac{L_{1}}{l_{\text {core }}}}}}-1\right)\left(e^{\frac{-x}{l_{\text {core }}}}-e^{\frac{-L_{1}}{l_{\text {core }}}}\right) \\
& -\left[\left(\rho_{0}+\frac{\rho}{T}\right) g+f_{w} \frac{\rho}{D_{h}}|u| u\right]\left(L_{1}-x\right)+P_{p}
\end{aligned}
$$

The same study can be done for all the other legs of the loop. The steps are not detailed but the method is easier and similar to above. There is neither a heat source nor sink along the horizontal legs containing the pressurizer and the pump (the temperature in the pressurizer is set to the temperature of the cells linked to it). The temperature and the pressure profiles along the pressurizer are: 


$$
\begin{aligned}
T_{\text {pressurizer }}(x) & =T_{\text {core }}\left(x=L_{1}\right) \\
P_{\text {pressurizer }}(x) & =P_{p}+f \frac{\rho_{0}}{D_{h}}|u| u\left(L_{1}+L_{3}-x\right) \text { for } x \in\left[L_{1} ; L_{1}+L_{3}\right] \\
P_{\text {pressurizer }}(x) & =P_{p} \text { for } x \in\left[L_{1}+L_{3} ; L_{1}+L_{3}+L_{4}\right] \\
P_{\text {pressurizer }}(x) & =P_{p}+f_{w} \frac{\rho_{0}}{D_{h}}|u| u\left(L_{1}+L_{3}+L_{4}-x\right) \\
& \text { for } x \in\left[L_{1}+L_{3}+L_{4} ; L_{1}+L_{2}\right]
\end{aligned}
$$

where $L_{3}$ and $L_{4}$ are the lengths given on Fig. 4.3 above, $P_{p}$ is the pressure in the pressurizer, $\rho_{0}$ the density and $D_{h}$ is the hydraulic diameter. At steadystate, the pressurizer does not exchange any mass and energy with the loop. As a result, the velocity $u_{p}$ defined in section 2.4 .2 , equals zero. This condition is important since it allows computing an analytical solution.

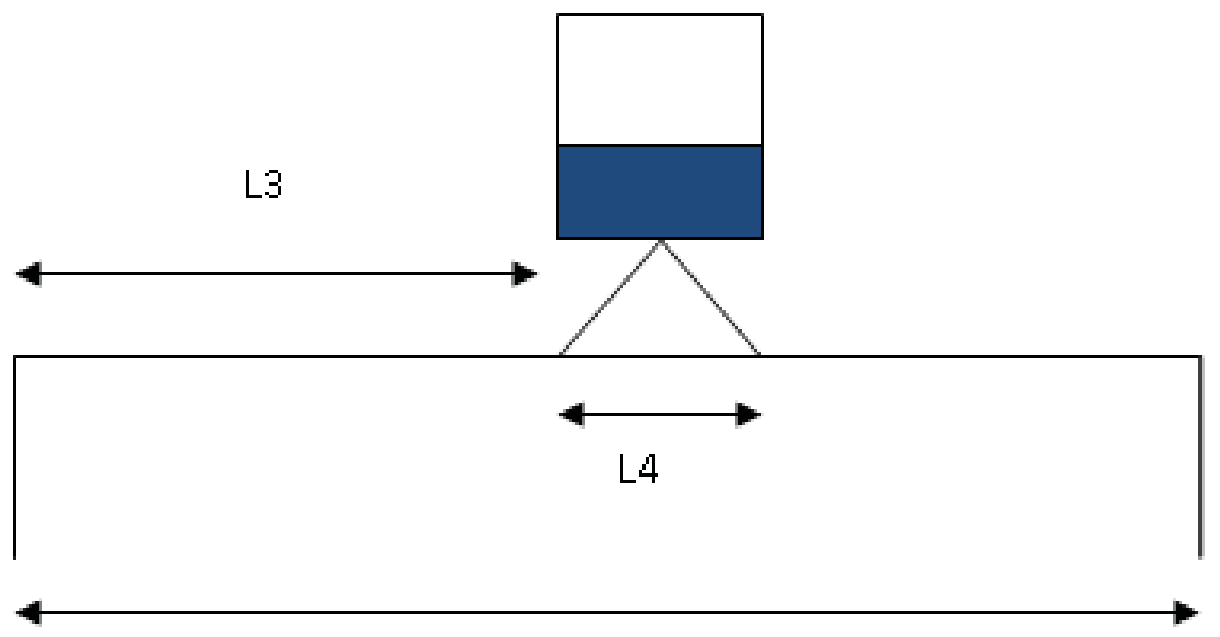

L2

Figure 4.3: Pressurizer

Along the pump, the temperature and pressure profiles are (Fig. 4.3 is a 
representation of the uniform pump over the length $L_{6}$ in the code) :

$$
\begin{gathered}
T_{\text {pump }}(x)=T_{s g}\left(x=2 L_{1}+L_{2}\right) \\
P_{\text {pump }}(x)=P_{s g}+f \frac{\rho_{0}}{D_{h}}|u| u\left(2 L_{1}+L_{2}-x\right) \text { for } x \in\left[2 L_{1}+L_{2} ; 2 L_{1}+L_{2}+L_{5}\right] \\
P_{\text {pump }}(x)=P_{s g}-L_{5} f_{w} \frac{\rho}{D_{h}}|u| u+\left(\rho g h-f_{w} \frac{\rho}{D_{h}}|u| u\right)\left(2 L_{1}+L_{2}+L_{5}-x\right) \\
\quad \text { for } x \in\left[2 L_{1}+L_{2}+L_{5} ; 2 L_{1}+L_{2}+L_{6}\right] \\
P_{\text {pump }}(x)=P_{s g}-\left(L_{5}+L_{6}\right) f_{w} \frac{\rho}{D_{h}}|u| u+f \frac{\rho_{0}}{D_{h}}|u| u\left(2 L_{1}+L_{2}+L_{5}-x\right) \\
\text { for } x \in\left[2 L_{1}+L_{2}+L_{6} ; 2 L_{1}+2 L_{2}\right]
\end{gathered}
$$

where $L_{5}$ and $L_{6}$ are lengths given on the figure Fig. 4.3. The temperature is constant in both horizontal legs of the loop. The pressure drop is due to the friction forces and the pump and varies linearly.

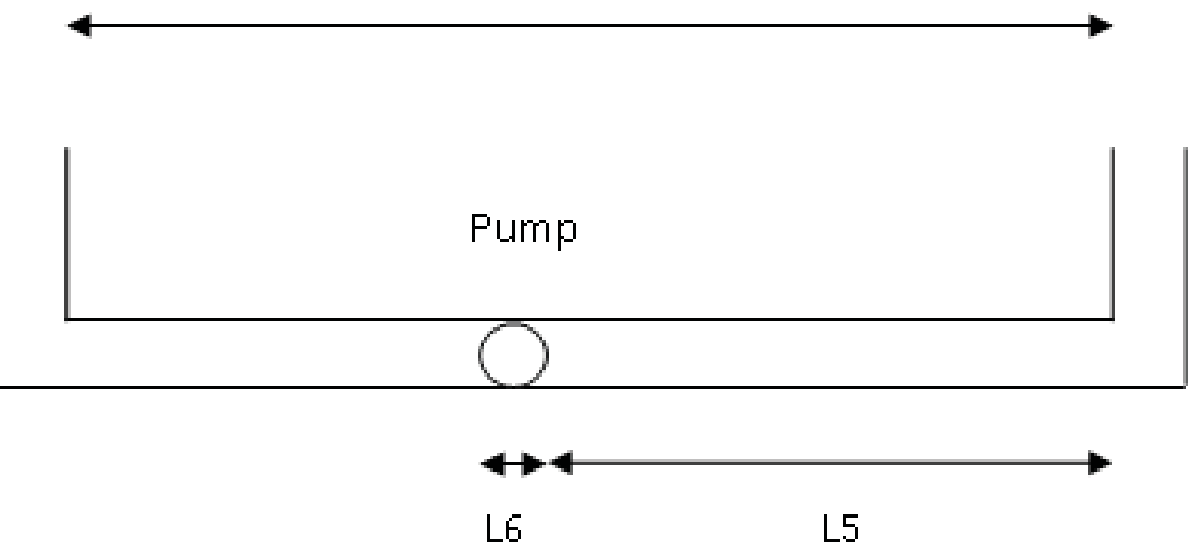

Figure 4.4: Pump

We notice above that the temperature profiles along the core and the steam generator are function of the parameters $l_{\text {core }}$ and $l_{s g}$ respectively, that also depend on the velocity. If the velocity is high (forced convection), the term $\frac{x}{l_{c o r e}}$ is small compare to one so that the exponential function can be simplified using a Taylor series development:

$$
e^{-x} \approx 1-x
$$

As a result, in the case of forced convection, the temperature profile is linear. If the buoyancy forces dominate (natural convection), the velocity is smaller than 
the previous case. The temperature profile will not be linear since the term $\frac{x}{l_{\text {core }}}$ is no longer small compare to one.

Knowing the analytical solution for the temperature and the pressure, a mesh refinement study can be performed. The spatial grid size is divided by two for each new simulations. The spatial grid size for the coarse grid is $\Delta x=2$ meters. The figure Fig. 4.5 shows the numerical solution for different grid sizes and the exact solution. Figure Fig. 4.6 shows the error versus space grid size.

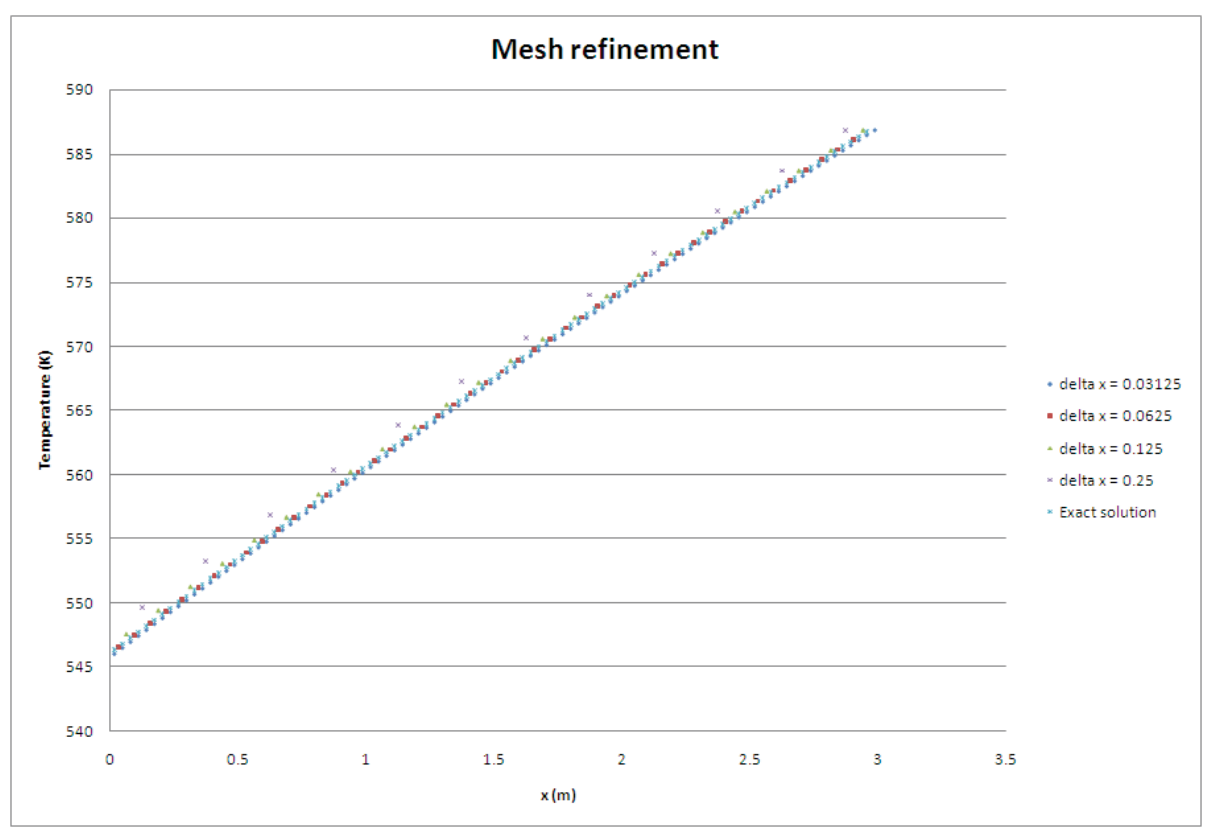

Figure 4.5: Mesh refinement 


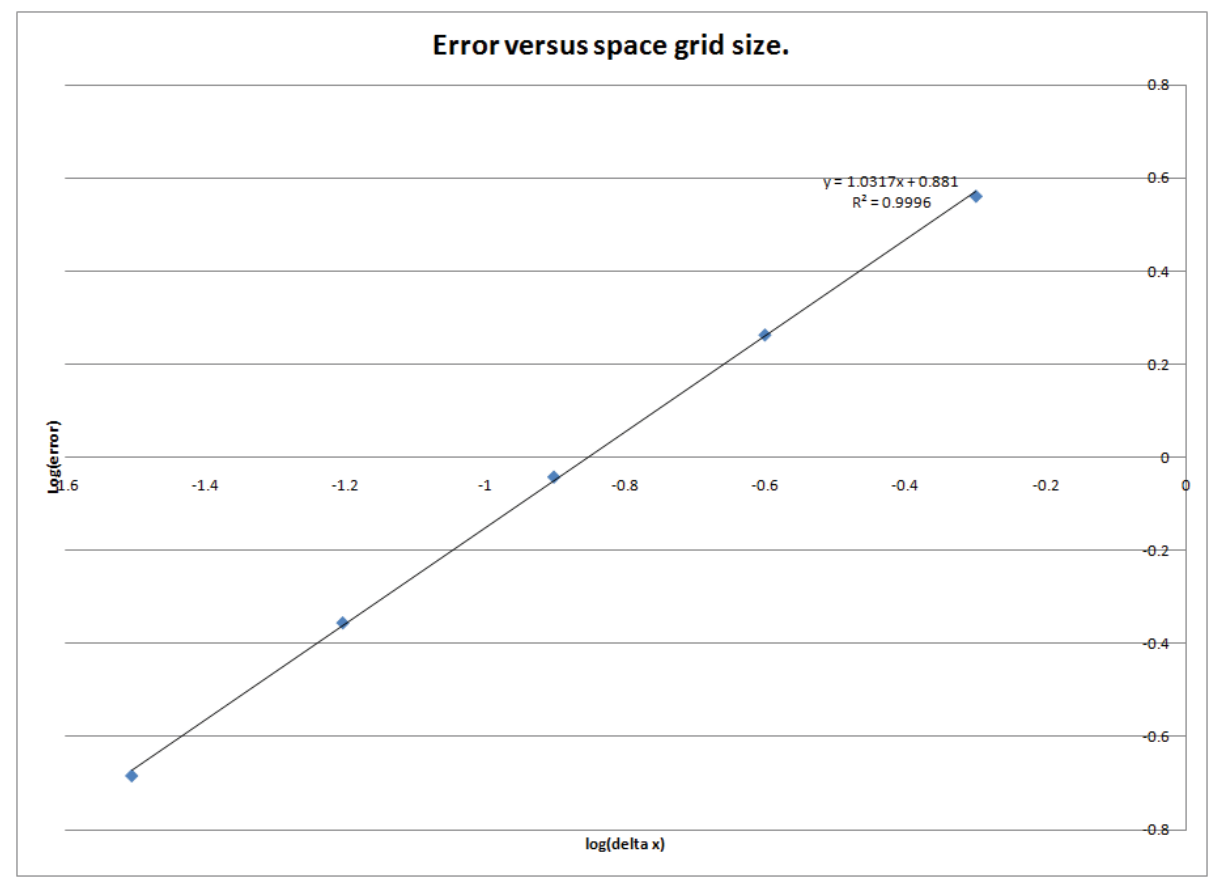

Figure 4.6: Error versus space grid size

As expected, as the mesh gets finer, the numerical solution converges to the exact solution (in yellow on Fig. 4.5). On Fig. 4.6 the slope of the straight line is 1.0317 as expected for a fist order code. 


\section{Chapter 5}

\section{Pressurized Water Reactor (PWR)}

This section deals with light water reactors. PWR characteristics are used in the model, and results obtained with compressible and incompressible models are provided in order to compare them at steady-state. Finally, a transient is simulated by turning the pump off in the system. A last section will deal with sensitivity analysis of the friction factor.

\subsection{Characteristic data for PWRs}

The simulation model possesses some input parameters which can be changed in order to match the flow data pertinent to the reactor under consideration. Here, we present the parameters employed for a PWR. The steady-state core inlet and outlet temperatures are $548 K$ and $588 K$ respectively. The velocity is of the order of $5 \mathrm{~m} \cdot \mathrm{s}^{-1}$. The Equation Of State is linearized at the following points: $P_{0}=15.5 \mathrm{MPa}$ and $T_{0}=569 \mathrm{~K}$. All physical parameters of the water are taken at this state point (e.g., $\rho_{0}$, viscosity, and heat capacity). The heat transfer coefficients are set in order to match the inlet and outlet temperatures in the core of $548 \mathrm{~K}$ and $588 \mathrm{~K}$ respectively. The pump strength is set to result in an average velocity of the order of $5 \mathrm{~m} . \mathrm{s}^{-1}$ in the loop. The core and steam generator temperatures are set to $T_{w c o r e}=800 \mathrm{~K}$ and $T_{w s g}=373 \mathrm{~K}$. All these inputs can be changed to match different types of PWR reactors.

\subsection{Steady-state and transient}

\subsubsection{Steady-state}

The steady-state is computed for the compressible and incompressible models and Darcy's law. We are interested both in the final steady-state and the tran- 
sient before the steady-state. A 9-second ramp for the heat source/sink is set. The initial conditions are atmospheric pressure and temperature. The initial velocity is $0.001 \mathrm{~m}^{-s^{-1}}$. The following plots Fig. 5.1, Fig. 5.2, Fig. 5.3 and Fig. 5.4 show the temperature, velocity, density and pressure profiles at steady-state and during the transient.

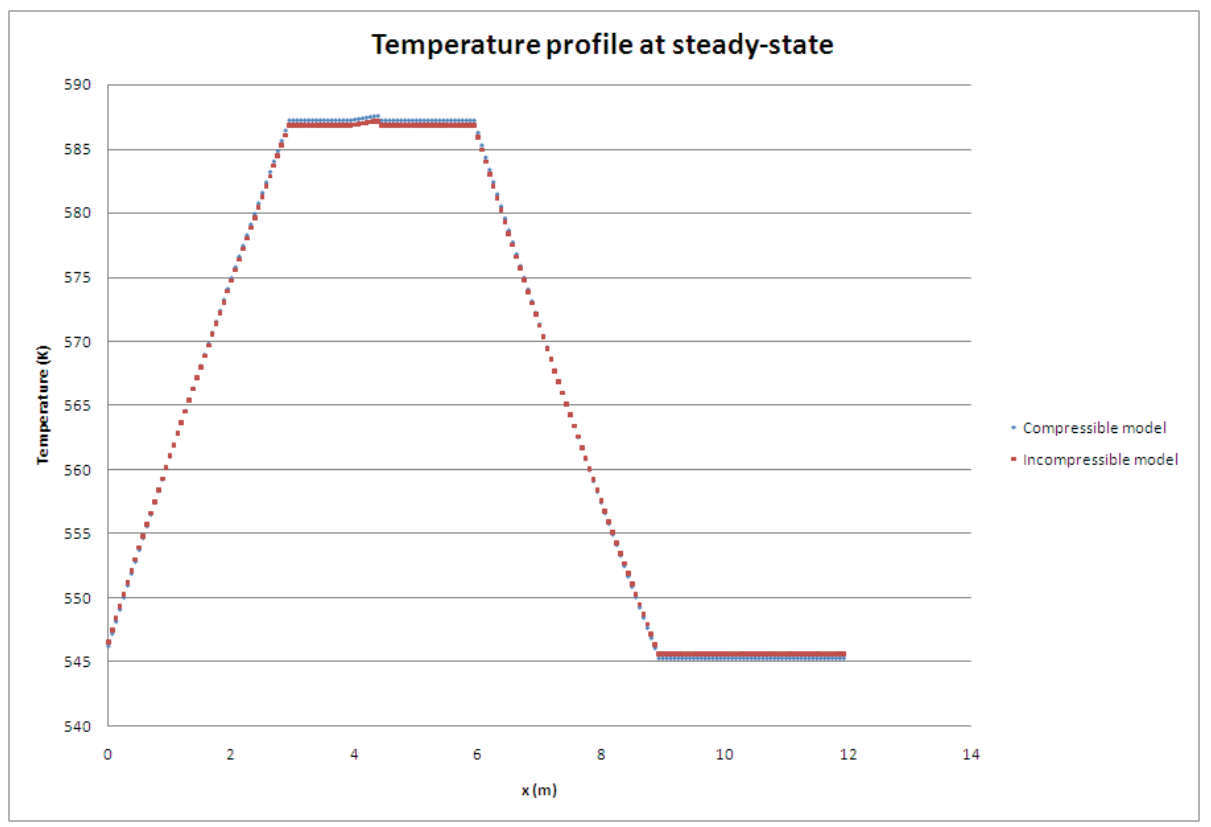

Figure 5.1: Temperature profile at steady-state

The temperature profile is linear along the core and the steam generator as explained above, and constant along the pressurizer and the pump. For the compressible model, the temperature is slightly higher than in the incompressible model. This offset is due to the dilatation of the fluid. 


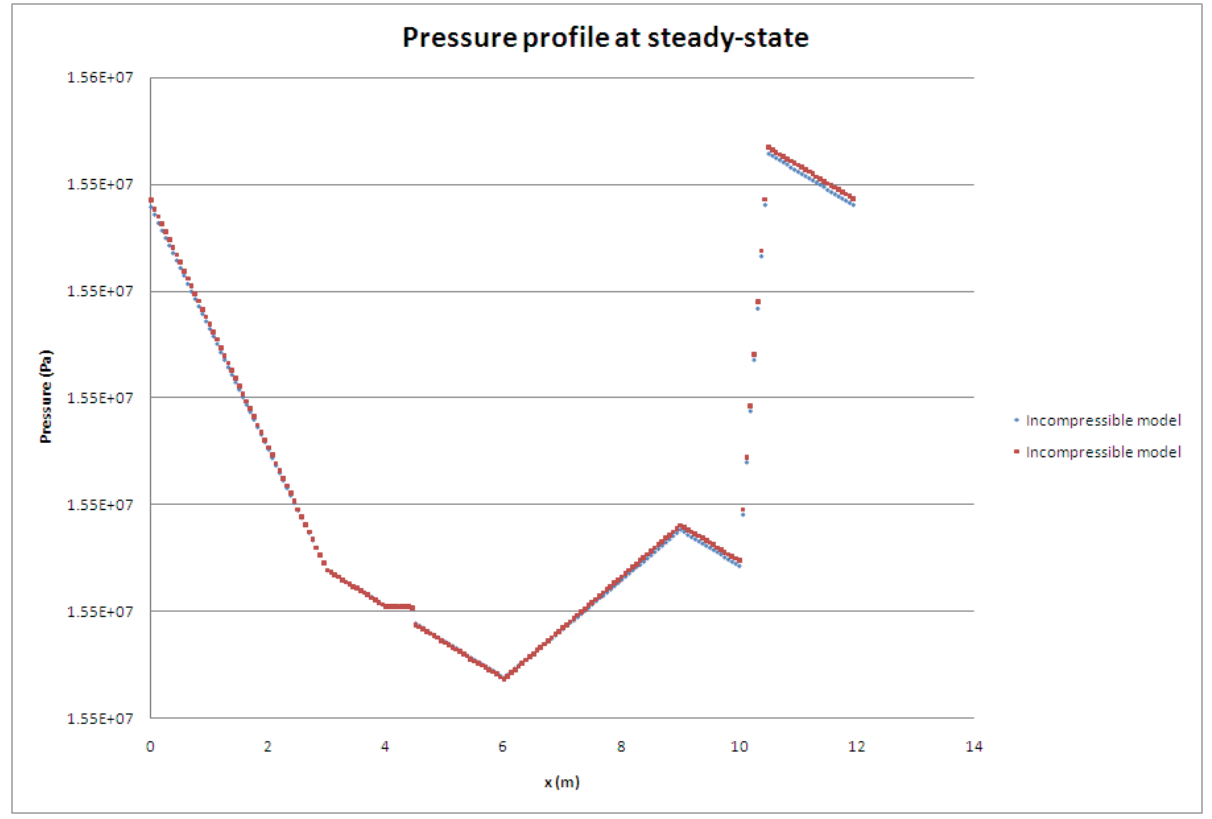

Figure 5.2: Pressure profile at steady-state

The pressure decreases along the core because of the gravity force and the increase in velocity. Along the upper leg, the pressurizer sets the pressure and a pressure drop is observed because of friction forces. Along the steam generator, gravity causes the pressure to rise. Fiction forces are not strong enough to cause a pressure drop. Along the lower leg, the pump adds an artificial pressure drop. 


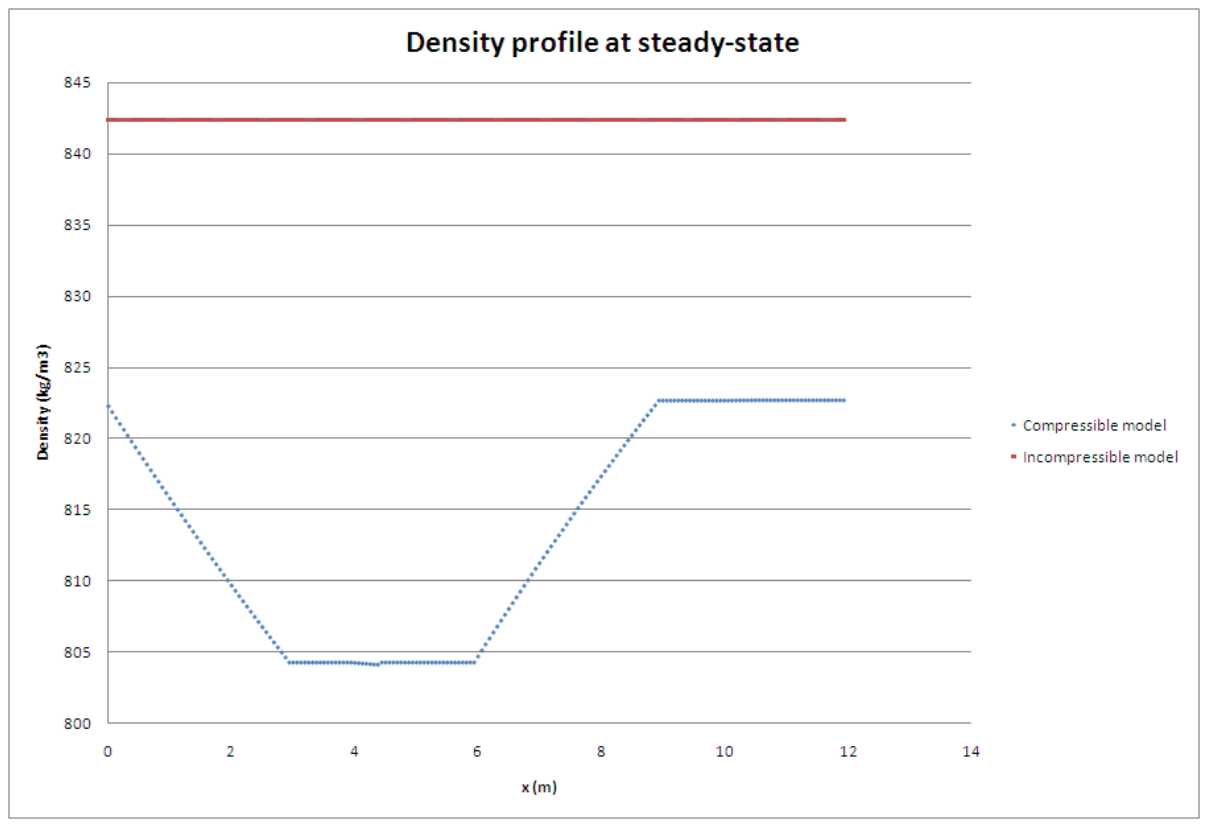

Figure 5.3: Density profile at steady-state

The density is coupled to the pressure and the temperature through the EOS. The pressure term in the EOS can be neglected in front of the temperature term since the sound speed is of the order of $10^{3}$. As a result, the density will be mainly sensitive to the change in temperature in the system. Then, it is expected to have a density gradient along the core and the steam generator. As the temperature rises, the density decreases. In the incompressible model the density is constant and higher than in the compressible model since it is not temperature-dependent. 


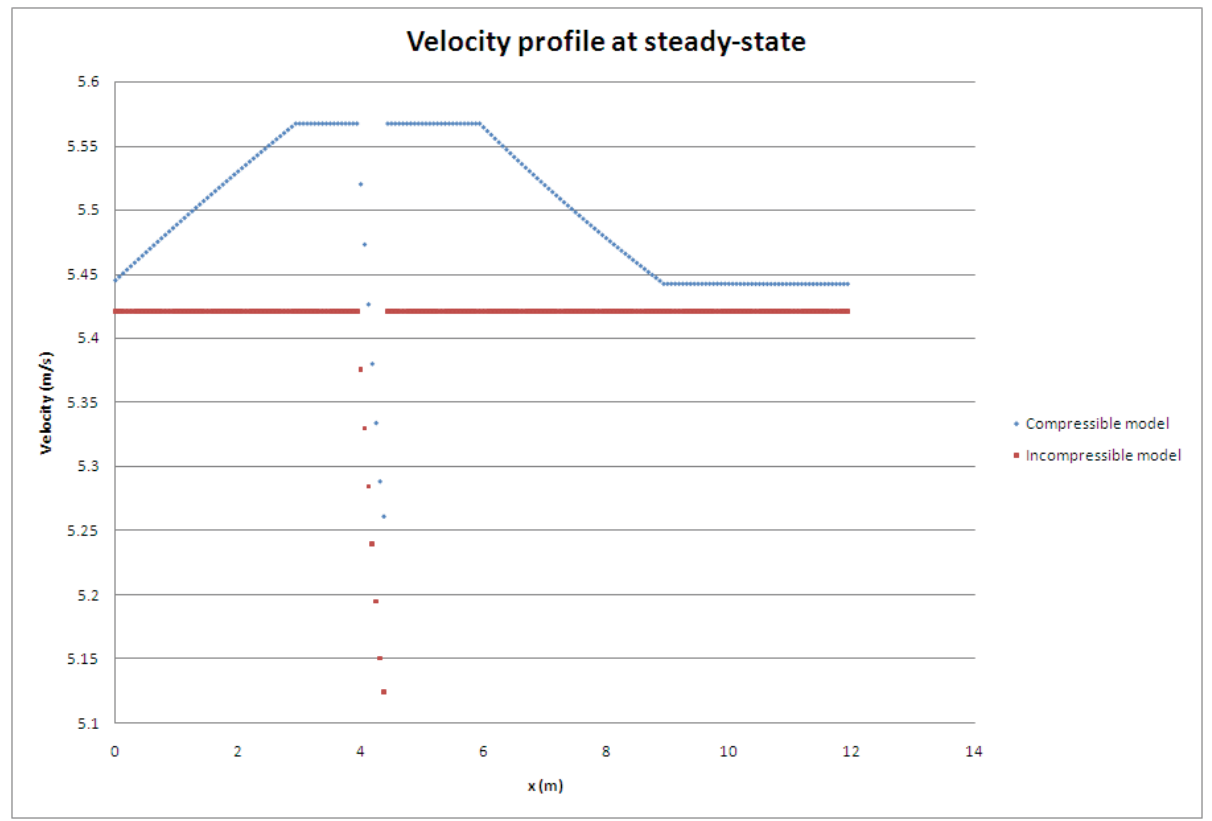

Figure 5.4: Velocity profile at steady-state

In the core, buoyancy forces make the velocity rise as temperature increases. The inverse phenomena is observed in the steam generator. Since the temperature is held constant in the pressurizer and pump legs, the velocity is also constant. In the case of the incompressible model, the continuity equation $\frac{\partial u}{\partial x}$ requires the velocity to be constant along the loop.

\subsubsection{Pseudo-transient to steady-state (with pumps on)}

This subsection aims at describing the transient to reach study-state as the pump is on. Let us recall that the purpose of this study is to quantify the uncertainty between models both at steady-state and during transient. The following plots Fig. 5.5, Fig. 5.6 and Fig. 5.7 show temperature, velocity and pressure variations during the transient. The last plot is the difference between the energy supplied to the fluid (in the core) and the energy removed from the fluid (in the steam generator). 


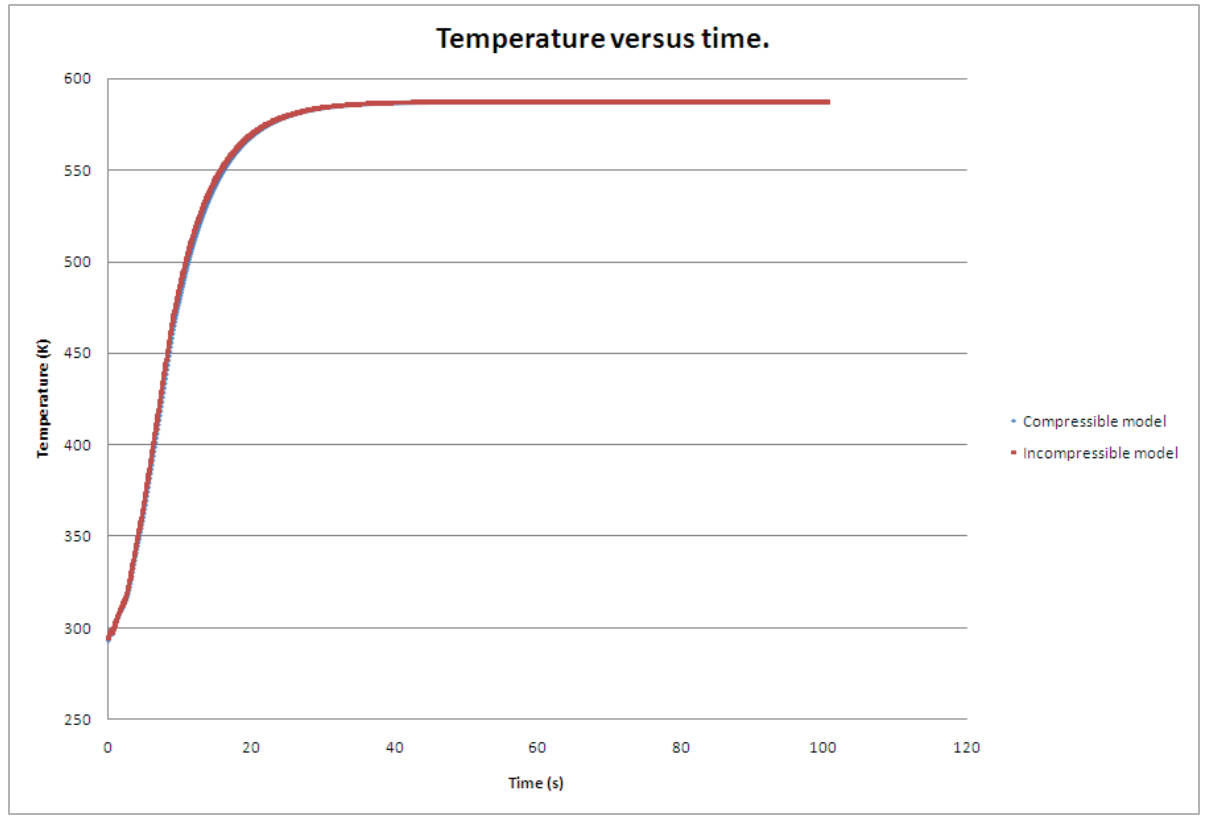

Figure 5.5: Temperature profile during the transient

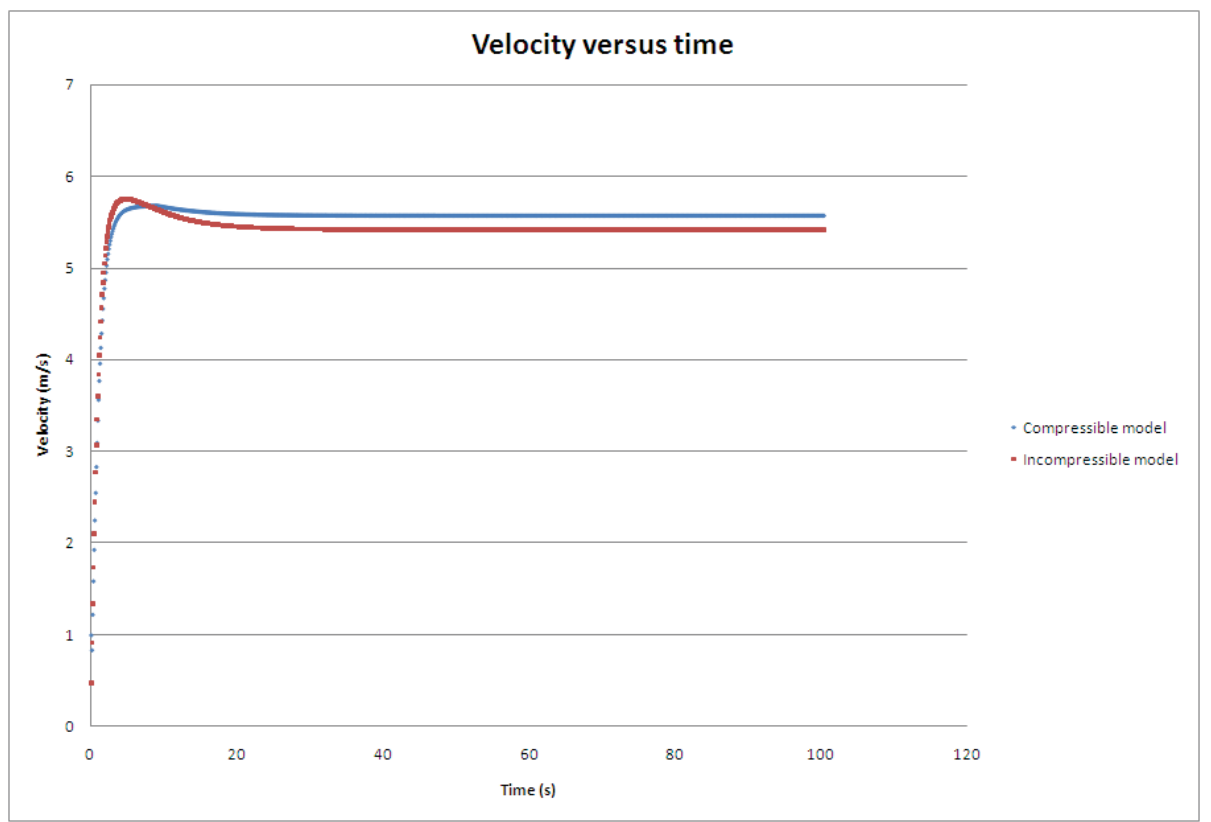

Figure 5.6: Velocity profile during the transient 
The velocity is a function of the pump strenght and reaches quickly a steadstate value. The steady-state is reached quicker with the incompressible model.

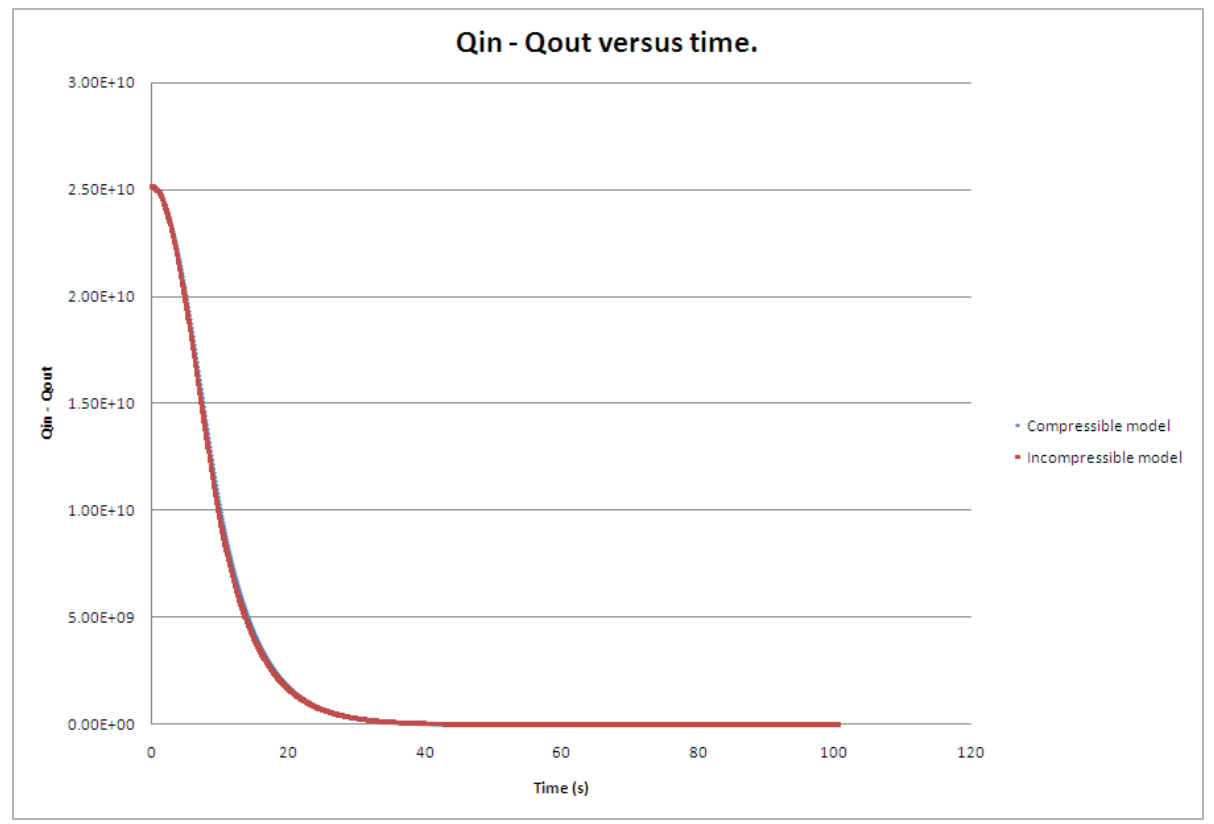

Figure 5.7: $Q_{\text {in }}-Q_{\text {out }}$ versus time

In order to reach steady-state, the system has to balance the energy removed from and supplied to the fluid. The initial temperature is set to $293 K$ so that the initial system is far from the steady-state. As a result, the term $Q_{i n}-$ $Q_{\text {out }}$ is large at the beginning of the transient and decreases afterwards. No major differences are found between the incompressible and compressible models during this pseudo transient.

\subsubsection{Model uncertainty}

The purpose of this study is the uncertainty quantification between compressible and incompressible models. In that regard, it is of interest to plot the change in velocity and density in the system in order to quantify importance of the term $\frac{\partial u}{\partial x}$. In the incompressible model, the continuity equation leads to $\frac{\partial u}{\partial x}=0$. More precisely, we want to compare $\frac{\partial u}{\partial x}$ to some terms of the momentum (for instance $\frac{\partial P}{\partial x}$ ) and energy equation (for instance $P \frac{\partial u}{\partial x}$ ), both at steady-state and during the transient. 


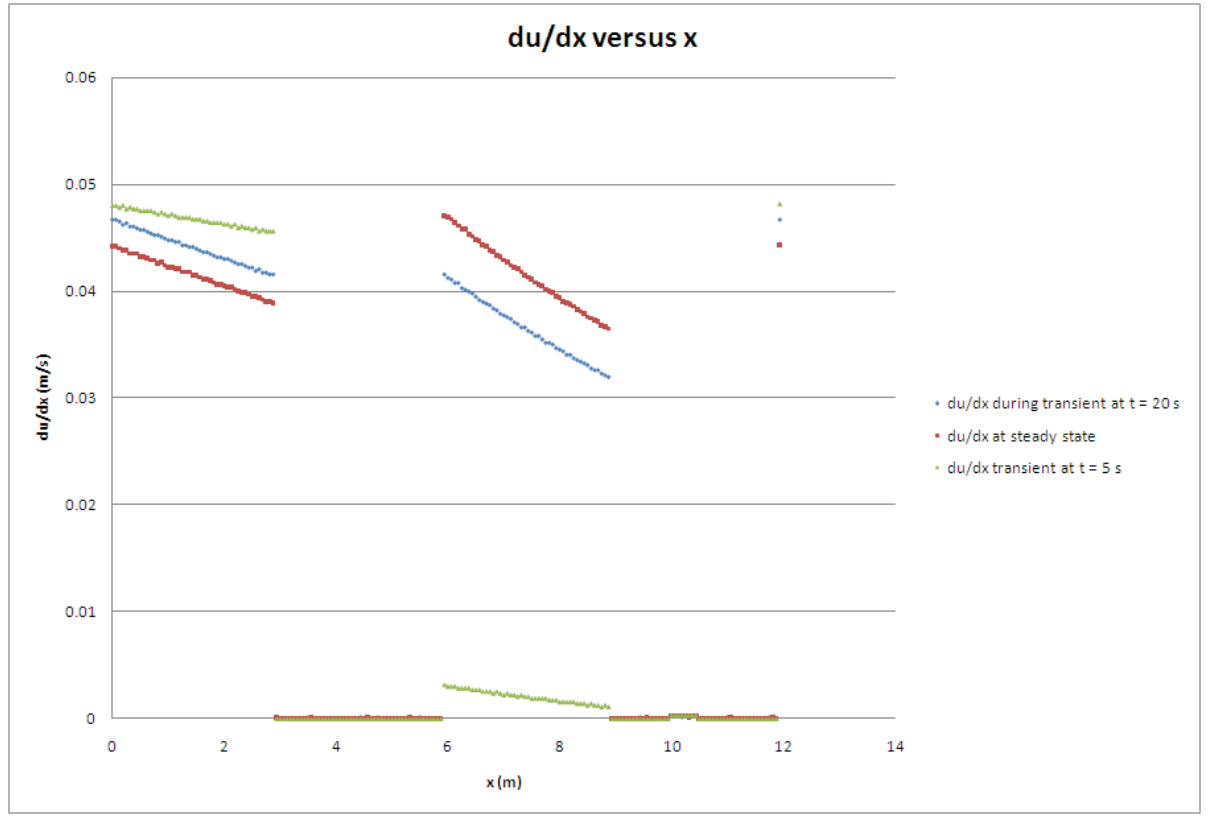

Figure 5.8: $\frac{\partial u}{\partial x}$ versus $\mathrm{x}$ at steady-state

From Fig. 5.8, one can conclude that the term $d u / d x$ is not larger than 0.05 in the system. Therefore, the assumption of an incompressible flow $(d u / d x=0)$ seems to be valid. 


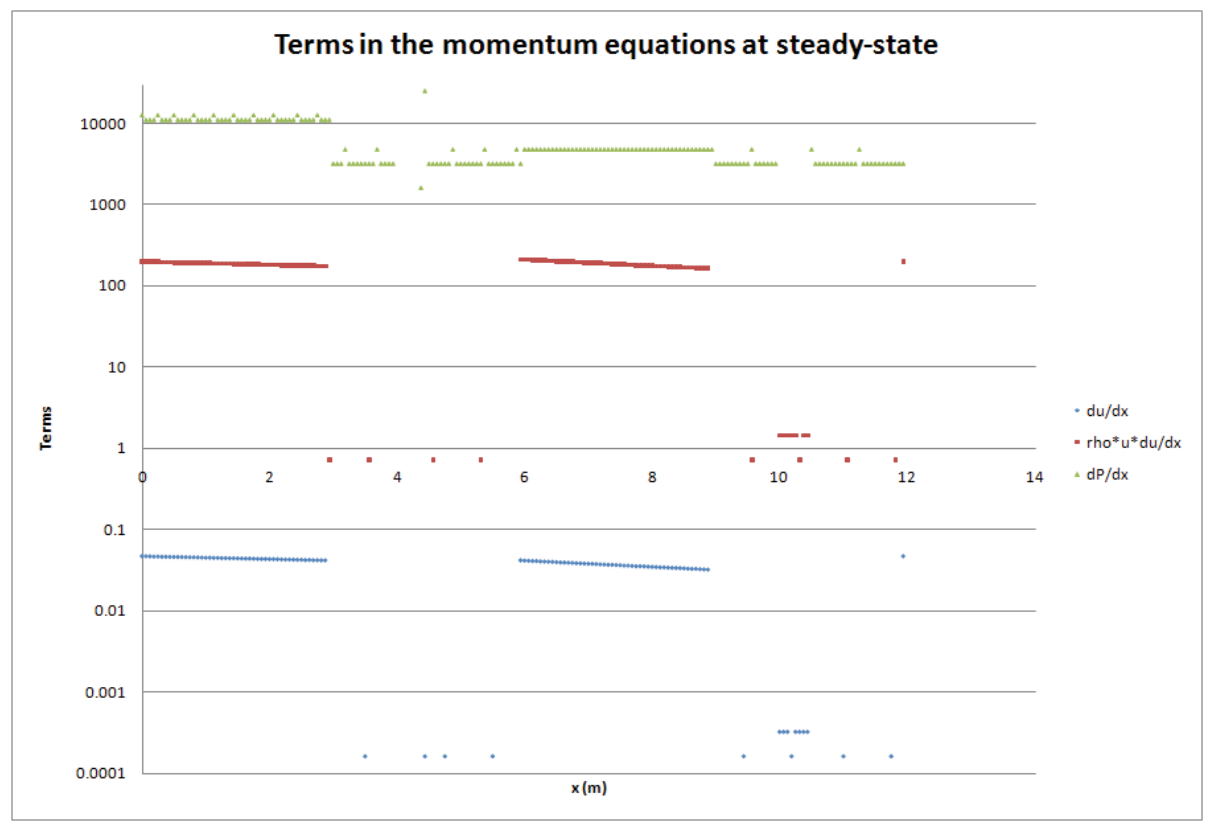

Figure 5.9: Terms in the momentum equation at steady-state versus $\mathrm{x}$

In Fig. 5.9, the magnitudes of different terms appearing in the momentum equation are compared (on a logarithm scale). It is obvious that the advection term, $\rho u \frac{\partial u}{\partial x}$ is the smallest, by about three orders of magnitude and then can be reasonably neglected for steady-state simulations. The same comparison was performed for the terms in energy equation and led to the same conclusions.

The change in velocity is within $2.5 \%$ the maximum and the minimum values. From the plots Fig. 5.8 and Fig. 5.9 above, the incompressible model seems to be a good correction when the term $\frac{\partial u}{\partial x}$ is small compared to the others terms. In addition, the temperature and pressure profiles are very similar during the transient with pumps on and at steady-state. In other terms, in the case of forced convection, the incompressible model seems to be a valid approximation to the compressible model in 1D.

\subsection{Study of a transient: pumps off}

The simulated transient consists in a pump coast-down, where the pump is completely stopped with a ramp of 4 seconds. In this case, buoyancy forces take over to cool down the core. Compressible-flow and incompressible-flow simulations are run with the same compressible steady-state values in order to start with the same amount of energy, mass and momentum. One question arises in doing so: what are the perturbations caused by the compressibleflow initial conditions when running the transient with the incompressible flow 
option? This question is addressed in Fig. 5.12 (green plot). The transient and the new steady-state (i.e., end of the transient) are given in the next sections.

\subsubsection{Transient: pumps off}

Fig. 5.10 and Fig. 5.11 show the variations of velocity and temperature as a function of time for the compressible and incompressible models.

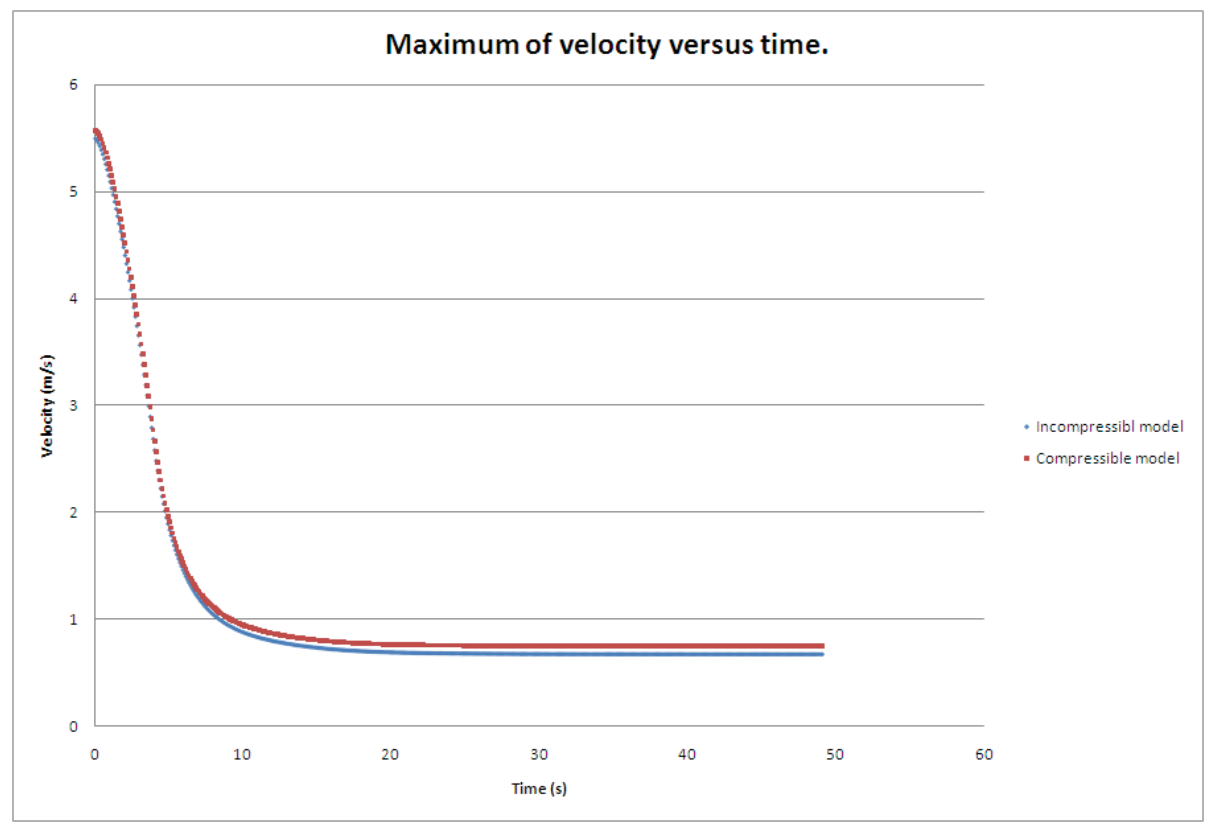

Figure 5.10: Maximum of velocity versus time during the transient

As the pump strength is decreased (but still not zero), the incompressible and compressible models yield the same velocity. As soon as the pump is fully off, buoyancy forces take over and a change in velocity is observed. Since the change in density is the motor of the buoyancy forces, the Boussinesq correction (density is temperature dependent as multiplied to the gravity and constant otherwise) is used in the incompressible model (for the compressible model, the density is pressure- and temperature-dependent) in order to account for the change in density. According to Fig. 5.10, the Boussinesq correction does not seem good enough for the incompressible model since the velocities are not the same between the two models on Fig. 5.10. 


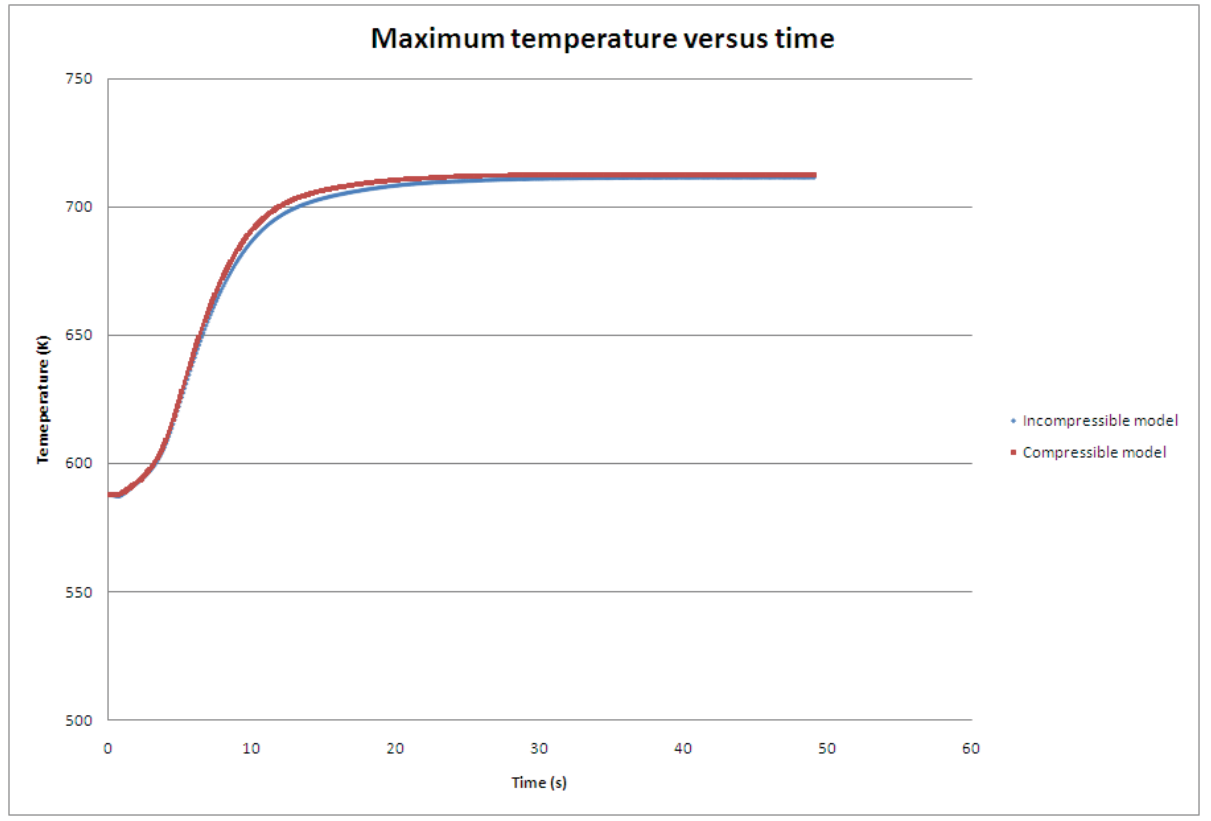

Figure 5.11: Maximum of temperature versus time during the transient

At the beginning and at the end (when the steady-state is reached) of the transient, the temperature profiles are identical. The main difference between the two models occurs when the temperature increase rapidly. As explained above, a change in velocity is observed; this leads to some modifications in the temperature since temperature and velocity are coupled to each other, i.e., when $\frac{d T}{d t}$ and $\frac{d u}{d t}$ are large. As it is shown in the section dealing with the mesh refinement, the temperature profile is coupled with velocity. 


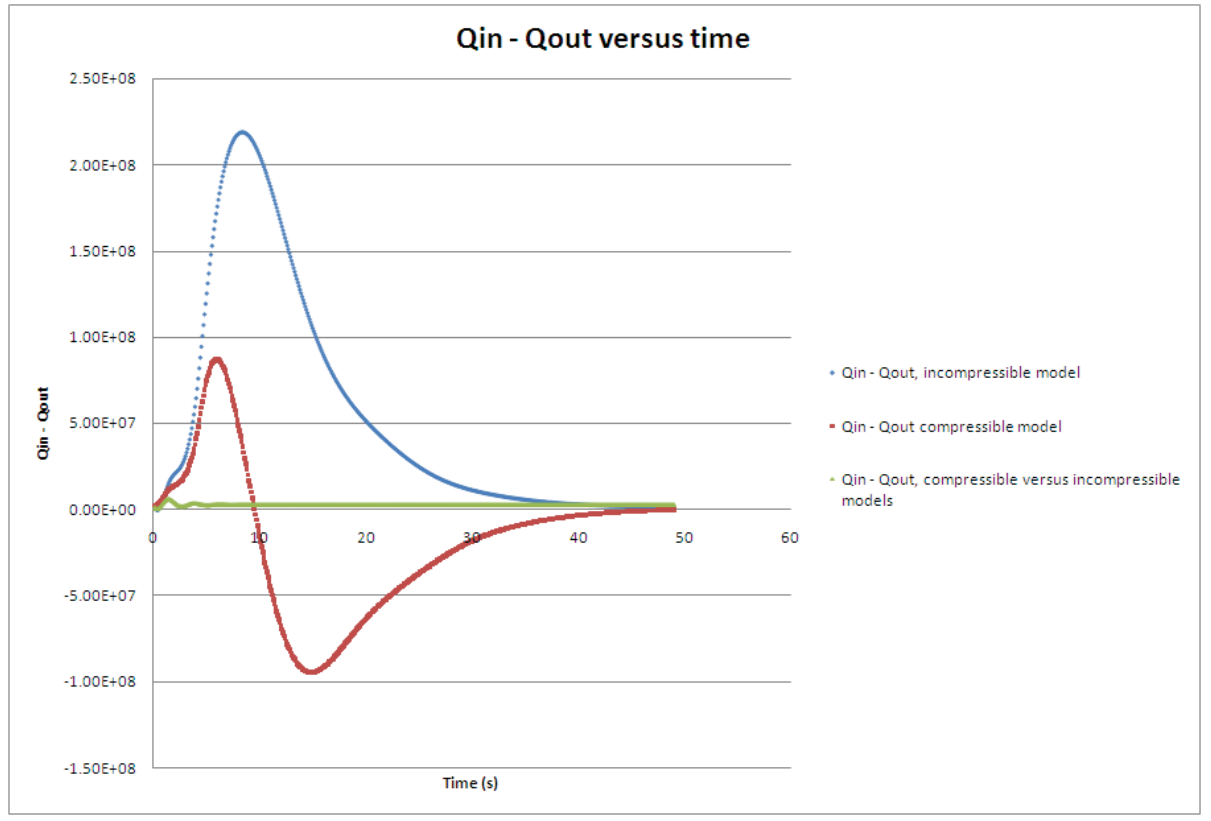

Figure 5.12: $Q_{\text {in }}-Q_{\text {out }}$ versus time during the transient

Fig. 5.12 shows that the energy removed from and supplied to the fluid strongly depends on the model. In the case of the incompressible model, this quantity is always positive, whereas for the compressible model, $Q_{\text {in }}-Q_{\text {out }}$ changes sign. It is observed that the same steady-state is reached. The third curve (green plot) in Fig. 5.12 presents the numerical solution of a compressible flow initial condition run with the incompressible option. The modifications (compressible to incompressible model) are much smaller than the variations due to the change in pump strength. As a result, it is considered that the modifications due to the impact of steady-state model are not important for this type of transient.

\subsubsection{Final steady-state after the transient}

In this section, the temperature, density and pressure profile are shown in Fig. 5.13, Fig. 5.14 and Fig. 5.15. 


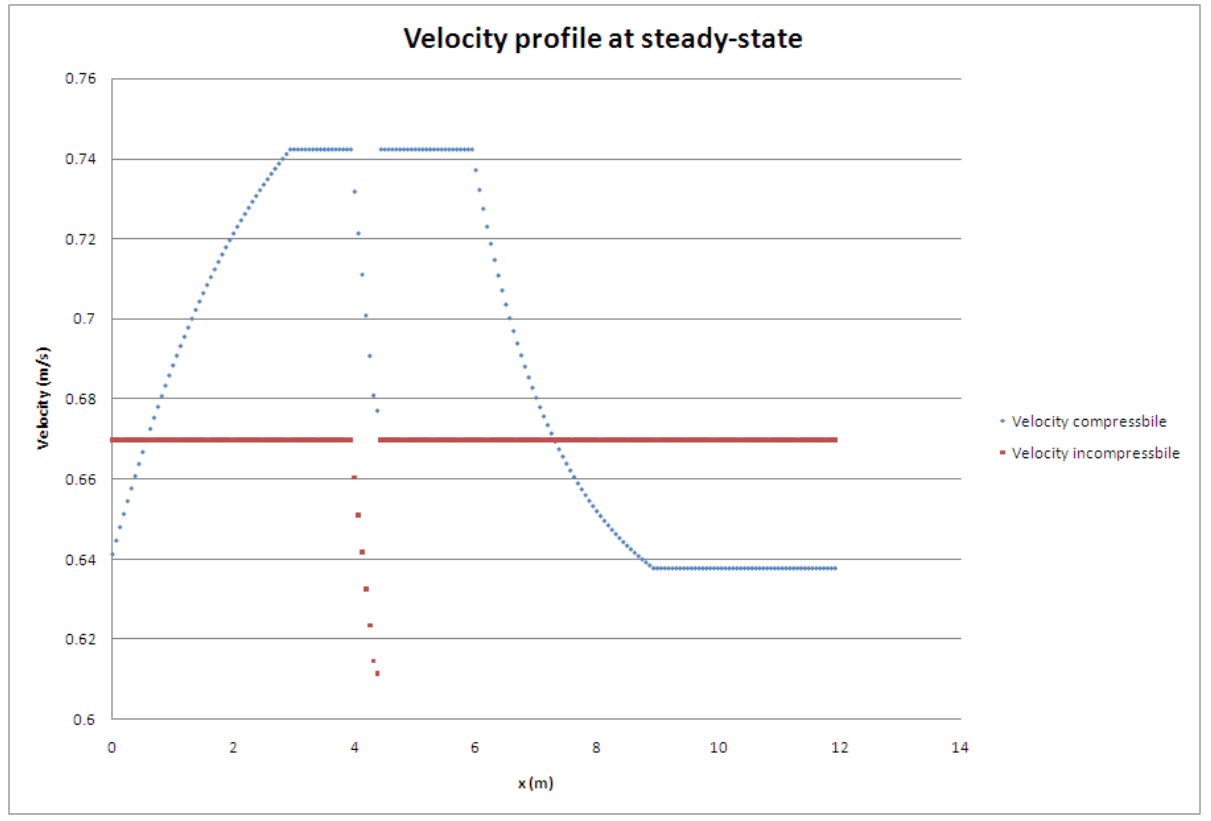

Figure 5.13: Velocity versus $\mathrm{x}$ after the transient

As expected, the velocity in the incompressible model is constant at steadystate since $\frac{d u}{d x}=0$ as shown in Fig. 5.13. In the compressible model, the velocity is function of space. Along the core, the fluid temperature rises so that the velocity will also increases because of buoyancy forces. Along the steam generator, the inverse phenomena is observed since the fluid is cooled down. Everywhere else, the fluid velocity is constant. The velocity experiences a change of almost $10 \%$ between the maximum and the minimum values that are larger than in the case of forced convection. It is noted a sharp drop at $x=4$ due to the pressurizer (the system is still not completely at steady-state). 


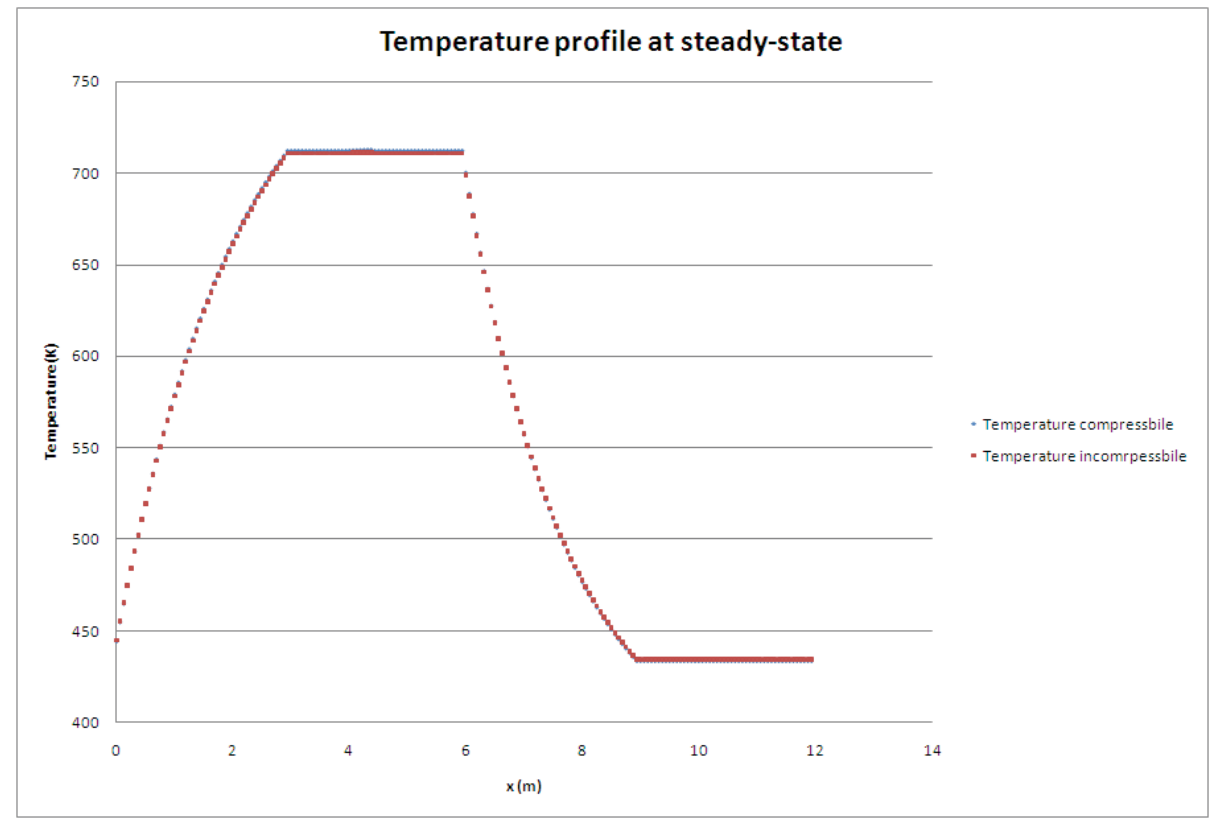

Figure 5.14: Temperature versus $\mathrm{x}$ after the transient

From Fig. 5.14, it is observed that the temperature profiles are similar to each other at steady-state. In addition, the shape matches the analytical solution computed in a previous section. It is noted that the length scale $l_{\text {core }}$ and $l_{s g}$ defined in Eq. (4.16) and Eq. (4.13) are proportional to the velocity that is smaller in the case of natural convection. Therefore, the exponential function can no longer be approximated by a linear function. 


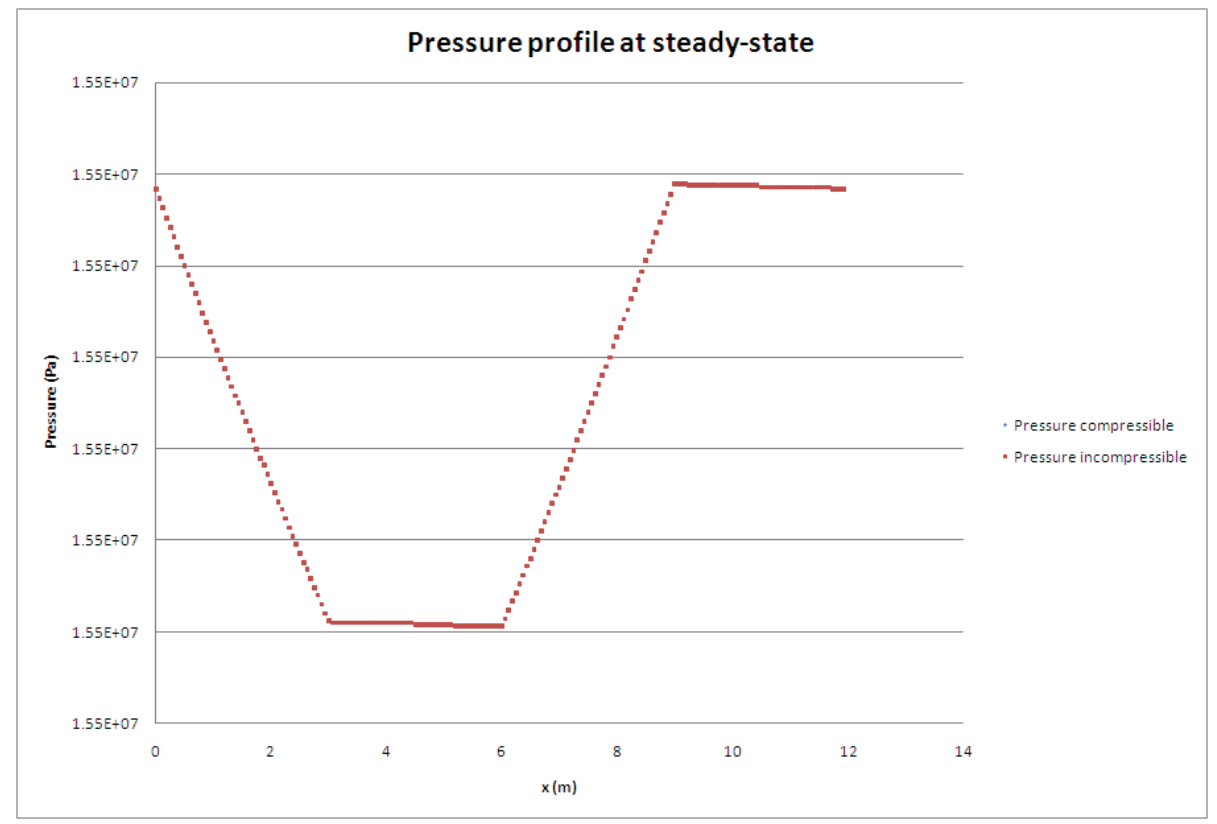

Figure 5.15: Pressure versus x after the transient

At steady-state and under natural convection conditions, the pressure forces are balanced by the gravity and friction forces. In the core and in the steam generator, the pressure gradient is mainly due to gravity forces. In the horizontal legs, the pressure drop is due to friction forces.

\subsubsection{Model sensitivity}

As in the previous section it is interesting to plot the term $\frac{d u}{d x}$ in order to see how accurate the incompressible assumption is. The following plots show the variations of the velocity at different dates during the transient (maximum of $\left.Q_{\text {in }}-Q_{\text {out }}\right)$ and at the final steady-state. 


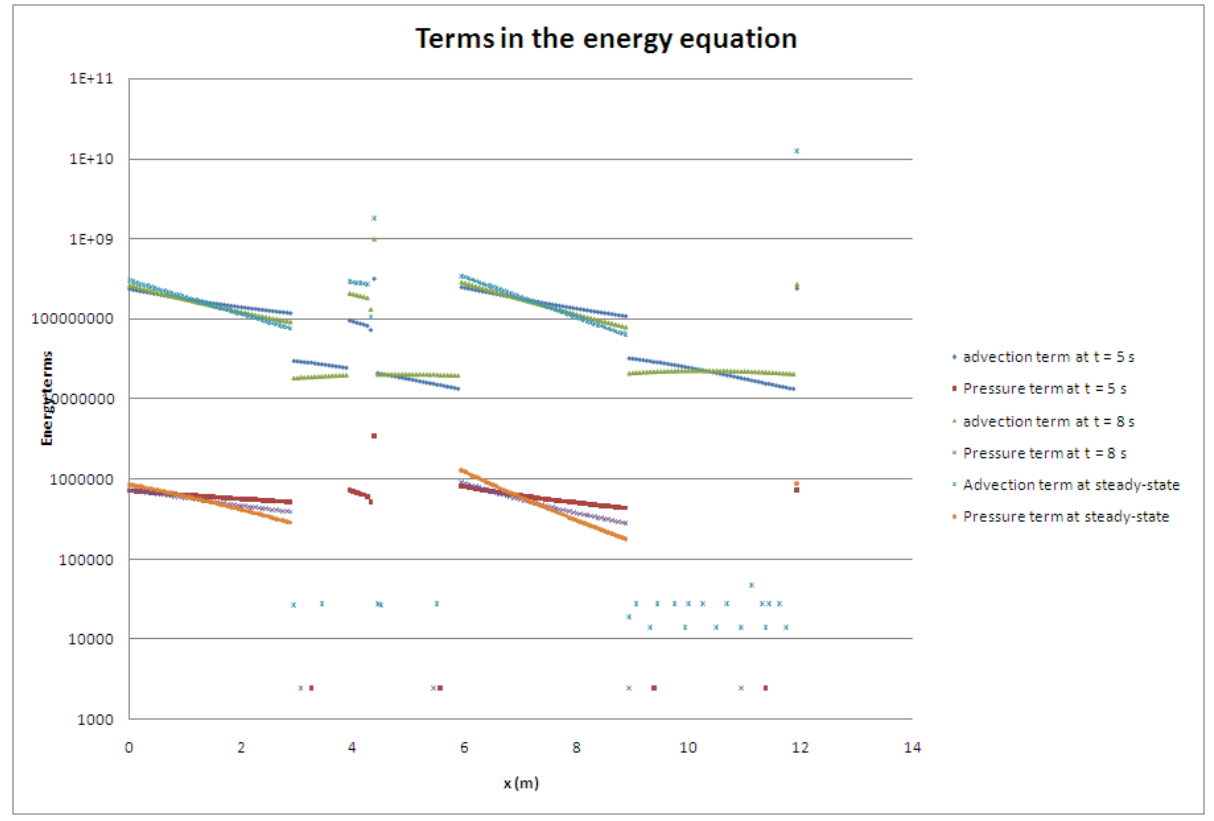

Figure 5.16: $Q_{\text {in }}-Q_{\text {out }}$ versus x at different time of the transient

From Fig. 5.16, it is clear that the advection term containing the term $\frac{d u}{d x}$ cannot be neglected in the energy equation. As a result, it seems that the incompressible model cannot be used as an approximation to the compressible model in the case of natural convection.

\subsection{Sensitivity analysis}

A sensitivity analysis is performed for the friction coefficient of the friction forces. This parameter normally comes from the experiment and is known with a large uncertainty. Three simulation are run with a variation of $\pm 5 \%$ of the friction parameter. We are mainly interested in the evolution of the plot $Q_{i n}-$ $Q_{\text {out }}$ as a function of time that are given in Fig. 5.17.

The Fig. 5.17 shows the variation of $\operatorname{frac}(\text { Qin }- \text { Qout })_{ \pm 5 \%}-($ Qin - Qout $)($ Qin - Qout $)$ versus time as the plot with the non-modified value of the friction parameter is taken as a reference. It is noted that the simulations seem very sensitive to modification of $5 \%$ of the friction factor. 


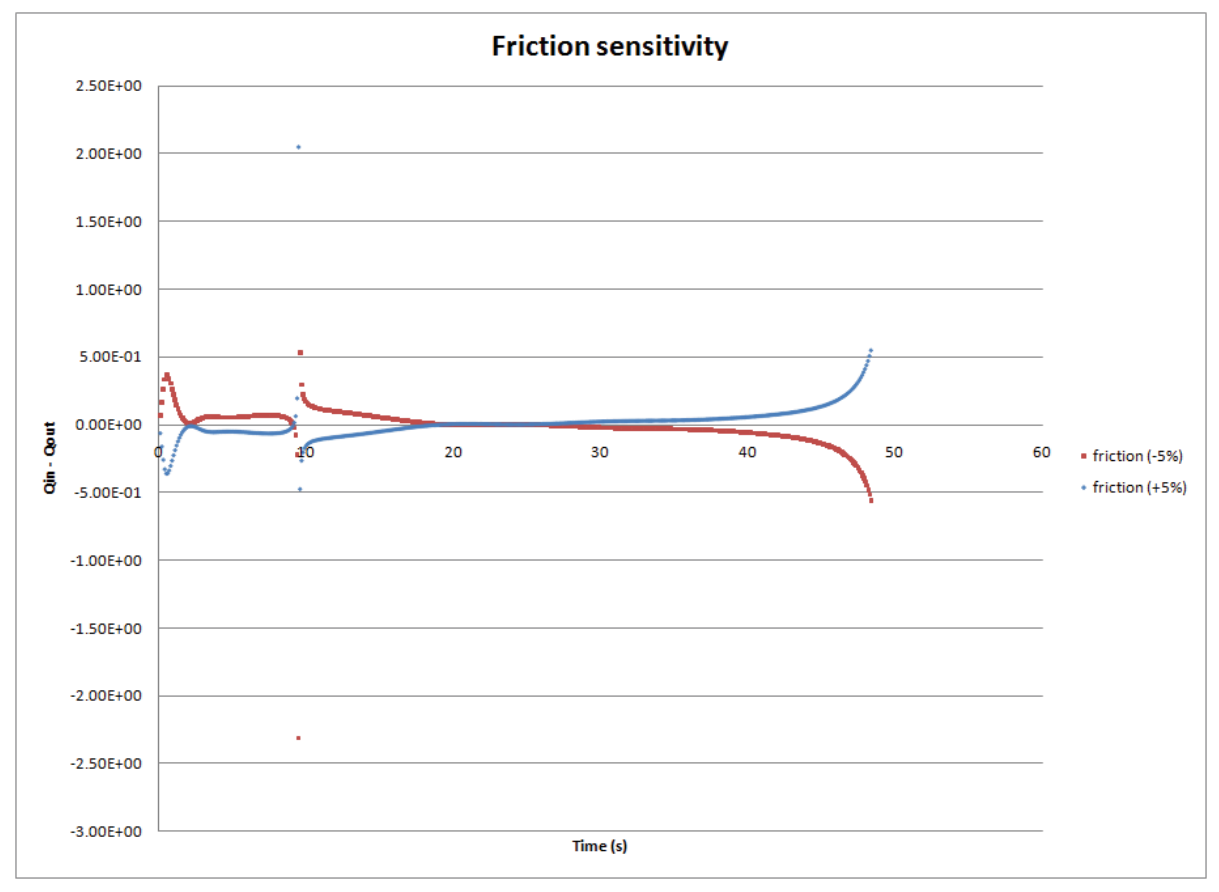

Figure 5.17: Friction sensitivity 


\section{Chapter 6}

\section{Conclusions and future work}

This study shows that the modified Euler Equations can be solved for the primitive variables with the Finite Volume method in 1D. The time and space convergence studies proves the code is first order in time and space. We also show that the code converges to the exact solution in the case of the incompressible model. The study of the transient and steady state with the characteristics of a PWR, allows determining when the incompressible model can be used.

- In forced convection and with a laminar flow, both models have the same transient and steady-state. The term $\frac{d u}{d x}$ can be neglected in this case.

- In natural convection, the incompressible model seems to be a bad approximation and does not give the same numerical solution as the compressible model. We show that the steady-state is still the same, but some strong changes occur during the transient.

In other terms, the compressible and incompressible models give the same numerical solution under forced convection and laminar flow, that is not the case for natural convection. Some future work and improvement might be done:

- The source term will include a feedback component $\frac{\partial Q}{\partial T}$, as well as a term to simulate a SCRAM.

- A correlation might be added for the heat transfer coefficients $h_{\text {core }}$ and $h_{s g}$.

- The friction term correlation will have to be changed in order to simulate High Temperature Gas Reactor. 


\section{Chapter 7}

\section{Appendix}

In the case of the incompressible model, it is possible to derive an analytical

solution from the equations. The modified Euler Equation in the case of the incompressible model are recalled:

$$
\begin{aligned}
\partial_{x}(u) & =0 \\
\rho \partial_{t} u+\rho u \partial_{x} u+\partial_{x} P+\hat{\rho} g\left(1-s_{p}\right)+f_{w} \frac{\rho}{D_{h}}|u| u & =0 \\
\rho C_{v} \partial_{t} T+\rho u C_{v} \partial_{x} T+P \partial_{x} u & =Q(x, t) \\
\rho & =\rho_{0} \\
\hat{\rho} & =\rho_{0}+\frac{\delta \rho}{\delta T}\left(T-T_{0}\right)
\end{aligned}
$$

here $\rho$ and hat $\rho^{\prime}$ are the constant density and the density computed with the Boussinesq correction, $u$ is the velocity, $T$ and $P$ are the temperature and pressure respectively, $f$ is the friction factor, $s_{p}$ is the pump strength, $D_{h}$ is the hydraulic diameter, $Q$ is the heat source and $C_{v}$ is the heat capacity. These equations contain a momentum source and energy source.

The momentum and energy equations can be simplified using the continuity equation:

$$
\begin{array}{r}
\rho_{0} \partial_{t} u+\partial_{x} P+\hat{\rho} g\left(1-s_{p}\right)+f \frac{\rho_{0}}{D_{h}}|u| u=0 \\
\rho_{0} C_{v} \partial_{t} T+\rho_{0} u C_{v} \partial_{x} T=Q(x, t)
\end{array}
$$

An analytical solution can be derived from the equation above by making the steady state assumption. The steps are detailed for the temperature and pressure profiles along the core and the results will be given for the other legs of the loop (pressurizer, steam generator and pump). Along the core, the heat source is $Q(x)=h_{\text {core }} L_{1}\left(T_{\text {wcore }}-T(x)\right)$ in one dimension where $h$ is the heat transfer coefficient, $L_{1}$ is the length of the core, $T_{w c o r e}$ is the wall temperature 
along the core and $T(x)$ is the profile of temperature along the core (it is assumed that the area is $L_{1} \times 1$. Then, the energy equation yields:

$$
\begin{array}{r}
\rho u C_{v} \partial_{x} T=h_{\text {core }} L_{1}\left(T_{\text {wcore }}-T(x)\right) \\
T(x)+\frac{\rho u C_{v}}{h_{\text {core }} L_{1}} T(x)=T_{\text {wcore }}
\end{array}
$$

This is a first order differential equation that can be solved analytically. To simplify the equation, lets define $l_{\text {core }}=\frac{\rho_{0} u C_{v}}{h_{c o r e} L_{1}}$ which is the length characteristic of the energy equation. The parameter $l_{\text {core }}$ depends on the fluid velocity so that it will affect the temperature profile as expected. A solution for this type of differential equation is (the temperature is solved for $x$ ):

$$
T(x)=A(x) e^{\frac{-x}{c o r e}}
$$

where $A$ is a function depending on the variable $x$ and will be computed using the differential equation as follows:

$$
\begin{array}{r}
A(x) e^{\frac{-x}{l_{\text {core }}}}+l_{\text {core }} \frac{\partial A(x)}{\partial x} e^{\frac{-x}{l_{\text {core }}}}-A(x) e^{\frac{-x}{l_{\text {core }}}}=T_{\text {wcore }} \\
l_{\text {core }} \frac{\partial A(x)}{\partial x} e^{\frac{-x}{l_{\text {core }}}}=T_{\text {wcore }} \\
\frac{\partial A(x)}{\partial x}=\frac{T_{\text {wcore }}}{l_{\text {core }}} e^{\frac{x}{l_{\text {core }}}}
\end{array}
$$

A differential equation for $A$ is obtained and can be solved:

$$
A(x)=T_{\text {core }} e^{\frac{x}{l_{\text {core }}}}+B
$$

where $B$ is a constant to determine afterwards using the boundary conditions. Then, the final solution is:

$$
T_{\text {core }}(x)=T_{\text {core }}+B e^{\frac{-x}{l_{\text {core }}}}
$$

This equation gives the temperature profile along the core. We can now derive the pressure profile along the core using the momentum equation. The temperature and the pressure are coupled through the Boussinesq correction in the momentum equation. At steady-state and along the core (no pump), the momentum equation yields:

$$
\begin{array}{r}
\partial_{x} P+\hat{\rho} g+f \frac{\rho}{D_{h}}|u| u=0 \\
\partial_{x} P+\left(\rho_{0}+\frac{\partial \rho}{\partial T}\left(T-T_{0}\right)\right) g+f \frac{\rho}{D_{h}}|u| u=0
\end{array}
$$

where $P$ and $T$ are the pressure and temperature respectively, $\hat{\rho}$ is the density computed using the Boussinesq correction, $\rho_{0}$ is the density at the linearization 
point, $f_{w}$ is the friction term, $g$ is the gravity term, $u$ is the velocity and $D_{h}$ is the hydraulic diameter. All parameters are constant but the temperature $T$ and the pressure $P$ that depend on space (not on time since this is steady-state). Using the solution for the temperature, a differential equation for the pressure can be derived as a function of space.

$$
\partial_{x} P+\left(\rho_{0}+\frac{\partial \rho}{\partial T}\left(T_{\text {core }}+B e^{\frac{-x}{l_{\text {core }}}}\right)-T_{0}\right) g+f \frac{\rho}{D_{h}}|u| u=0
$$

It remains to integrate over space from $x=0$ to $x=L_{1}$ where $L_{1}$ is the length of the core.

$$
P(x)=\frac{B g}{l_{\text {core }}} e^{\frac{-x}{l}}-\left[\left(\rho_{0}+\frac{\rho}{T}\right) g-f_{w} \frac{\rho}{D_{h}}|u| u\right] x+C
$$

where $\mathrm{C}$ is a constant to determine using the boundary condition. We will assume that the pressurizer is right after the core so that the pressure is fixed in $x=L_{1}$.

$$
P(x)=\frac{B g}{l_{\text {core }}}\left(e^{\frac{-x}{l_{\text {core }}}}-e^{\frac{-L_{1}}{l_{\text {core }}}}\right)-\left[\left(\rho_{0}+\frac{\rho}{T}\right) g+f_{w} \frac{\rho}{D_{h}}|u| u\right]\left(L_{1}-x\right)+P_{p}
$$

where $P_{p}$ is the pressure in the pressurizer. This equation gives the pressure as a function of space at steady-state along the core. This analytical solution will be used as a reference for the numerical solution. Of course, the constant $B$ has to be computed. This requires to compute the temperature profile along the steam generator. The energy equation to solve for the steam generator is the following:

$$
\begin{array}{r}
\rho u C_{v} \partial_{x} T=h_{s g} L_{1}\left(T_{w s g}-T(x)\right) \\
T(x)+\frac{\rho u C_{v}}{h_{s g} L_{1}} T(x)=T_{w s g}
\end{array}
$$

where $T_{w s g}$ is the temperature in the steam generator, $h_{s g}$ is the heat transfer coefficient between the fluid and the steam generator. All other variables are defined as above. We also define $l_{s g}=\frac{\rho u C_{v}}{h_{s g} L_{1}}$ as the space scale of the energy equation along the steam generator. The method of solving is the same as above so that the solution is:

$$
T_{s g}(x)=T_{w s g}+D e^{\frac{-x}{l_{s g}}}
$$

where $D$ is a constant. To determine the constants $B$ and $D$, two equations are required. The first one is obtained by saying that the temperature along the pump is constant, since in this model, the pump does not modify the energy equation (this assumption is not true along the pressurizer). As a result: 


$$
\begin{aligned}
T_{\text {core }}(x=0) & =T_{s g}\left(x=2 L_{1}+L_{2}\right) \\
T_{w c o r e}+B & =T_{w s g}+D e^{\frac{-\left(2 L_{1}+L_{2}\right)}{l_{s g}}}
\end{aligned}
$$

where $B$ and $D$ are the constants to compute, $T_{s g}$ and $T_{\text {core }}$ are the temperature in the steam generator and in the core respectively, and $L_{1}$ and $L_{2}$ are the loop lengths.

The second equation is obtained by considering that the steady-state is reached when the energy put into and removed from the system equal each other:

$$
\begin{aligned}
Q_{\text {in }} & =-Q_{\text {out }} \\
\int_{0}^{L_{1}} h_{\text {core }} L_{1}\left(T_{\text {core }}(x)-T_{\text {core }}\right) & =-\int_{L_{1}+L_{2}}^{2 L_{1}+L_{2}} h_{\text {sg }} L_{1}\left(T_{\text {sg }}(x)-T_{\text {sg }} \gamma 7\right.
\end{aligned}
$$

where $L_{1}$ and $L_{2}$ are the lengths of the loop, $h_{\text {core }}, h_{s g}, T_{\text {core }}$ and $T_{s g}$ are the heat transfer coefficients for the core and the steam generator, and the temperature for the core and steam generator respectively. By integrating this equation, a second relation between $B$ and $D$ is derived. Using these two relations above, the system can be solved and the constants expressed as a function of the boundary conditions (since the system is periodic, the boundary conditions are defined as the temperature in the core and in the stem generator).

$$
\begin{gathered}
D=\frac{T_{\text {wcore }}-T_{w s g}}{e^{-\frac{\left(2 L_{1}+L_{2}\right)}{l_{s g}}}-\frac{l_{s g} h_{s g}}{l_{\text {core }} h_{\text {core }}} \frac{e^{-\frac{\left(2 L_{1}+L_{2}\right)}{l_{s g}}}-e^{-\frac{\left(L_{1}+L_{2}\right)}{l_{s g}}}}{1-e^{-\frac{L_{1}}{l_{\text {core }}}}}} \\
B=\left(T_{\text {wcore }}-T_{w s g}\right)\left(\frac{1}{1-\frac{l_{s g} h_{s g}}{l_{\text {core }} h_{\text {core }}} \frac{1-e^{-\frac{L_{1}}{l_{s g}}}}{1-e^{-\frac{L_{1}}{l_{\text {core }}}}}-1}\right)
\end{gathered}
$$

As it can be seen above, the constants $B$ and $D$ are independent of the initial conditions. As a result, the numerical solution is unique and does not depend on the initial conditions, in the case of the incompressible model (this was verified). Since the constants $B$ and $D$ are known, the pressure equations can be derived:

$$
\begin{aligned}
& P(x)=\frac{\left(T_{w c o r e}-T_{w s g}\right) g}{l_{\text {core }}}\left(\frac{1}{1-\frac{l_{s g} h_{s g}}{l_{\text {core }} h_{\text {core }}} \frac{1-e^{-\frac{L_{1}}{l_{s g}}}}{1-e^{-\frac{L_{1}}{l_{\text {core }}}}}}-1\right)\left(e^{\frac{-x}{l_{\text {core }}}}-e^{\frac{-L_{1}}{l_{\text {core }}}}\right) \\
& -\left[\left(\rho_{0}+\frac{\rho}{T}\right) g+f_{w} \frac{\rho}{D_{h}}|u| u\right]\left(L_{1}-x\right)+P_{p}
\end{aligned}
$$


The same study can be done for all the other legs of the loop. The steps are not detailed but the method is easier and similar to above. There is neither heat source nor sink along the horizontal legs containing the pressurizer and the pump (the temperature in the pressurizer is set to the temperature of the cells linked to it). The temperature and the pressure profiles along the pressurizer are:

$$
\begin{aligned}
T_{\text {pressurizer }}(x) & =T_{\text {core }}\left(x=L_{1}\right) \\
P_{\text {pressurizer }}(x) & =P_{p}+f \frac{\rho_{0}}{D_{h}}|u| u\left(L_{1}+L_{3}-x\right) \text { for } x \in\left[L_{1} ; L_{1}+L_{3}\right] \\
P_{\text {pressurizer }}(x) & =P_{p} \text { for } x \in\left[L_{1}+L_{3} ; L_{1}+L_{3}+L_{4}\right] \\
P_{\text {pressurizer }}(x) & =P_{p}+f_{w} \frac{\rho_{0}}{D_{h}}|u| u\left(L_{1}+L_{3}+L_{4}-x\right) \\
& \quad \text { for } x \in\left[L_{1}+L_{3}+L_{4} ; L_{1}+L_{2}\right]
\end{aligned}
$$

where $L_{3}$ and $L_{4}$ are the lengths given on the figure Fig. 4.3 above, $P_{p}$ is the pressure in the pressurizer, $\rho_{0}$ the density and $D_{h}$ is the hydraulic diameter. At steady-state, the pressurizer does not exchange any mass and energy with the loop. As a result, the velocity $u_{p}$ defined in a previous section, equals zero. This condition is important since it allows computing an analytical solution.

$$
\begin{gathered}
T_{\text {pump }}(x)=T_{s g}\left(x=2 L_{1}+L_{2}\right) \\
P_{\text {pump }}(x)=P_{s g}+f \frac{\rho_{0}}{D_{h}}|u| u\left(2 L_{1}+L_{2}-x\right) \text { for } x \in\left[2 L_{1}+L_{2} ; 2 L_{1}+L_{2}+L_{5}\right] \\
P_{\text {pump }}(x)=P_{s g}-L_{5} f_{w} \frac{\rho}{D_{h}}|u| u+\left(\rho g h-f_{w} \frac{\rho}{D_{h}}|u| u\right)\left(2 L_{1}+L_{2}+L_{5}-x\right) \\
\quad \text { for } x \in\left[2 L_{1}+L_{2}+L_{5} ; 2 L_{1}+L_{2}+L_{6}\right] \\
P_{\text {pump }}(x)=P_{s g}-\left(L_{5}+L_{6}\right) f_{w} \frac{\rho}{D_{h}}|u| u+f \frac{\rho_{0}}{D_{h}}|u| u\left(2 L_{1}+L_{2}+L_{5}-x\right) \\
\text { for } x \in\left[2 L_{1}+L_{2}+L_{6} ; 2 L_{1}+2 L_{2}\right]
\end{gathered}
$$

where $L_{5}$ and $L_{6}$ are lengths given on the figure Fig. 4.3. The temperature is constant in both horizontal legs of the loop. The pressure drop is due to the friction forces and the pump and varies linearly.

We notice above that the temperature profiles along the core and the steam generator are function of the parameters $l_{\text {core }}$ and $l_{s g}$ respectively, that also depend on the velocity. If the velocity is high (forced convection), the term $\frac{x}{l_{\text {core }}}$ is small compare to one so that the exponential function can be simplified using a Taylor series development:

$$
e^{-x} \approx 1-x
$$

As a result, in case of forced convection, the temperature profile is linear. If the buoyancy forces dominate (natural convection), the velocity is smaller than the 
previous case. The temperature profile will not be linear since the term $\frac{x}{l_{\text {core }}}$ is no longer small compare to one. 


\section{Bibliography}

[1] H. Fenech ,

"Heat Transfer and Fluid Flow in Nuclear System",

Pergamon Press, 1981

[2] C.A.J. Fletcher, "Computational Techniques for Fluid Dynamics",

Springer-Verlag, pp. 296-298, 1990

[3] J.D. Ramshaw and V. Mousseau,

"Damped artificial compressibility method for steady-state low-speed flow calculations" ,

Computers Fluids, Vol. 20, No. 2, pp.177-186(1991) 